\title{
Cryptic species delineation in freshwater planarians of the genus Dugesia (Platyhelminthes, Tricladida): Extreme intraindividual genetic diversity, morphological stasis, and karyological variability
}

\author{
Laia Leria ${ }^{\mathrm{a}}$, Miquel Vila-Farréb, Marta Álvarez-Presas ${ }^{\mathrm{a}}$, Alejandro Sánchez-Gracia ${ }^{\mathrm{a}}$, Julio Rozas ${ }^{\mathrm{a}}$, \\ Ronald Sluys $^{\mathrm{c}}$, Marta Riutort ${ }^{\mathrm{a}, *}$ \\ ${ }^{a}$ Dpt. de Genètica, Microbiologia i Estadística, Facultat de Biologia, and Institut de Recerca de la Biodiversitat (IRBio), Universitat de Barcelona, Barcelona, Catalonia, \\ Spain \\ ${ }^{\mathrm{b}}$ Max Planck Institute of Molecular Cell Biology and Genetics, Dresden, Germany \\ ${ }^{\mathrm{c}}$ Naturalis Biodiversity Center, P.O. Box 9517, 2300 RA Leiden, the Netherlands
}

\section{A R T I C L E I N F O}

\section{Keywords:}

ABGD

BPP

GMYC

Integrative taxonomy

Mosaicism

mPTP

\begin{abstract}
A B S T R A C T
The keystone of planarian taxonomy traditionally has been the anatomy of the copulatory apparatus. However, many planarian species comprise asexual fissiparous populations, with the fissiparous animals not developing a copulatory apparatus, thus precluding their morphological identification. Incorporation of molecular data into planarian systematics has been of great value, not only in the identification of fissiparous individuals but also as an additional source of information for determining species boundaries. Nevertheless, the discrepancy between morphological and molecular data has highlighted the need for extra sources of taxonomic information. Moreover, a recent study has pointed out that fissiparous reproduction may lead to high levels of intraindividual genetic diversity in planarians, which may mislead molecular analyses. In the present study we aim to test a new up-to-date integrative taxonomic procedure for planarians, including intraindividual genetic data and additional sources of taxonomic information, besides morphology and DNA, using Dugesia subtentaculata sensu lato as a model organism, a species with an intricate taxonomic history. First, we used three different methods for molecular species delimitation on single locus datasets, both with and without intraindividual information, for formulating Primary Species Hypotheses (PSHs). Subsequently, Secondary Species Hypotheses (SSHs) were formulated on the basis of three types of information: (1) a coalescent-based species delimitation method applied to multilocus data, (2) morphology of the copulatory apparatus, and (3) karyological metrics. This resulted in the delimitation of four morphologically cryptic species within the nominal species $D$. subtentaculata. Our results provide evidence that the analysis of intraindividual genetic data is essential for properly developing PSHs in planarians. Our study reveals also that karyological differentiation, rather than morphological differentiation, may play an important role in speciation processes in planarians, thus suggesting that the currently known diversity of the group could be highly underestimated.
\end{abstract}

\section{Introduction}

Discovering and describing species is not only important from a taxonomic point of view, but also because species are the fundamental units for other disciplines, such as ecology and conservation biology. However, defining and recognizing species is challenging, and during the history of systematic biology many different species concepts have been formulated (e.g., biological, ecological, phenetic, and phylogenetic concepts) (de Queiroz, 2007; Sluys and Hazevoet, 1999). Presently, a conceptual agreement is emerging among biologists in which species are considered as independently evolving metapopulation lineages, being known as the General Lineage Species Concept (de Queiroz, 1998). Under this conceptual framework, other species concepts, such as, for example, the biological and phylogenetic species concepts are considered to be species recognition criteria, instead of concepts, that are used as different lines of evidence to delineate independently evolving lineages (de Queiroz, 2007; Frankham et al., 2012).

Although systematic studies may be based on only one of these lines of evidence for species delimitation, it has now become customary to

\footnotetext{
* Corresponding author.

E-mail address: mriutort@ub.edu (M. Riutort).
} 
search for congruence between several types of information, thus applying a practical method currently known under the term Integrative Taxonomy (Dayrat, 2005; Padial and Miralles, 2010; Schlick-Steiner et al., 2010). One of the most frequently used integrative taxonomic procedures consists of formulating Primary Species Hypotheses (PSHs), (i.e., hypotheses on candidate species on the basis of a single type of information, in general a single-locus molecular approach), followed by the evaluation of these PSHs with the help of other kinds of evidence, which then leads to the formulation of Secondary Species Hypotheses (SSHs) and the consequent taxonomic decisions (Pante et al., 2015a). Among the different criteria used to establish SSHs, the ones used most often concern morphology and multilocus DNA sequence data (Pante et al., 2015b). In case of congruence between these lines of evidence, confidence for the SSHs is high, which, subsequently, may be formalized through the description of new species. In case of non-congruence, additional sources of information, besides DNA and morphology, have been proven highly useful, not only for increasing confidence in the SSHs but also for inferring the putative causes underlying such incongruences between morphological and molecular evolution (Dejaco et al., 2016).

One of the most common cases of incongruence between morphological and molecular data concerns cryptic species. Cryptic species are defined as lineages that independently diversified but retained the same morphological characteristics. Basically, cryptic species have the same taxonomic status as species, albeit that the morphological information that is usually applied for recognition fails to discriminate these taxa. The notion of cryptic speciation was suggested a considerable time ago (Mayr, 1942), but it has not been until recently that the number of reported cryptic species has greatly increased, presently including examples from most animal phyla (Pérez-Ponce de León and Poulin, 2016). Different evolutionary mechanisms have been proposed to explain cryptic speciation, such as morphological stasis, convergence, or recent diversification (Struck et al., 2018). Unfortunately, a high proportion of these cryptic species remains undescribed, which precludes the integration of this important part of biodiversity into different fields of research (Fišer et al., 2018).

The increasing use of molecular data in systematic studies has promoted the development of several approaches for molecular species delimitation (Ence and Carstens, 2011; Pons et al., 2006; Puillandre et al., 2012; Yang and Rannala, 2010; Zhang et al., 2013). These methods can be classified under two principal categories, viz. validation and discovery methods, reflecting the condition whether the samples need to be partitioned a priori or not, respectively. These methods also differ in the type of input data that is used (genetic distances, phylogenetic trees, or allele sharing) and in the number of loci that can be incorporated (Flot, 2015), which may lead to some discrepancies between the delimitations obtained with the various methods (Carstens et al., 2013, Luo et al., 2018). Discovery methods can work with singlelocus data and are generally used to formulate PSHs, while the validation methods that use information from multiple loci are generally used to develop SSHs (e.g., Razkin et al., 2017; Van Steenkiste et al., 2018).

Although initially only mitochondrial data had been widely used in species delimitation approaches, principally due to its high rate of sequence evolution, it has been shown that inclusion of multiple nuclear loci can help to detect putative genetic processes of introgression or incomplete lineage sorting, which, when present, may be reflected in the species hypotheses (Knowles and Carstens, 2007; Dejaco et al., 2016; Obertegger et al., 2018; Papakostas et al., 2016). Therefore, when including molecular data in a systematic study, it is important not only to apply different methods for species delimitation but also to use several mitochondrial and nuclear loci.

Species delimitation in free-living freshwater flatworms of the genus Dugesia Girard, 1850 (Platyhelminthes, Tricladida, Dugesiidae) traditionally has been based mostly on morphological data, concerning the anatomy of the copulatory apparatus, occasionally supplemented with karyological data. However, many Dugesia species may reproduce asexually by fission, with the fissiparous animals not developing a copulatory apparatus, thus precluding their morphological identification. In these cases, incorporation of molecular data has been of great value, not only in the identification of asexual individuals of known species (Lázaro et al., 2009) but also in tracing new species boundaries in Dugesia (Sluys et al., 2013). However, the only gene that has been used so far for species delimitation in this genus is the mitochondrial gene Cytochrome c oxidase I (Cox1).

Features such as geographic distribution, mode of reproduction, and karyology are sometimes reported in Dugesia species descriptions as extra characteristics of the species, but generally these data are not used as diagnostic characters. Different geographical distribution among lineages generally should not be used as a diagnostic trait of their evolutionary independence because of the possibility of changes in their distribution, either due to natural events (e.g., dispersion or extinction) or to human-mediated translocations (Pongratz et al., 2003; Solà, 2014). Similarly, although information on reproductive strategy may be very informative for detecting speciation processes, it is known that individuals of many Dugesia species are able to alternate between reproductive strategies (Stocchino and Manconi, 2013; and references therein). Thus, in this genus, information on reproductive strategy may not constitute a reliable source of evidence for tracing species boundaries. In contrast, differences in ploidy level and centromeric position of the chromosomes have been reported for different Dugesia species (Pala et al., 1999; Ribas, 1990). Furthermore, several Dugesia species have been described with a chromosome portrait that differs from the most common haploid complement of $\mathrm{n}=8$, exhibiting complements such as $\mathrm{n}=7$ or $\mathrm{n}=9$ (Ball, 1970; Gourbault, 1981; Kawakatsu et al., 1976; Pala et al., 1981; Stocchino et al., 2004). Therefore, karyological data may be a very informative additional line of evidence to be included in systematic studies of this genus.

The focal species of our study, Dugesia subtentaculata (Draparnaud, 1801), inhabits the Western Mediterranean region, with a total of 13 known localities scattered in Southern France, the Iberian Peninsula, Northern Africa, and the Balearic Islands (De Vries, 1986a; De Vries, 1988a; Lázaro et al., 2009). Dugesia subtentaculata was first described from near Montpellier (France) as being oviparous in spring and fissiparous in summer (Draparnaud, 1801). Some years later, the sexually reproducing specimens were assigned to the species Dugesia gonocephala (Dugès, 1830), while the fissiparous individuals remained as D. subtentaculata. However, in 1925, after a morphological re-examination of the two species, it was concluded that they were conspecific, and therefore the junior synonym ( $D$. gonocephala) was assigned to the specimens, due to the difficulty of identifying the asexual individuals (Vandel, 1925). A good number of years later the new species Dugesia iberica Gourbault \& Benazzi, 1979 was described from Mallorca and the Iberian Peninsula; this new species was externally and anatomically similar to D. gonocephala (Gourbault and Benazzi, 1979). Finally, in a taxonomic revision of these three species it was concluded that (1) $D$. subtentaculata is a different species than $D$. gonocephala, and (2) that $D$. iberica is conspecific with $D$. subtentaculata, the latter being the junior and valid species name (De Vries, 1986a). Thus, the separate taxonomic status of $D$. subtentaculata was re-established.

A few years later, in a karyological study on freshwater flatworms of the Iberian Peninsula, certain populations of $D$. subtentaculata showed differences in ploidy level, centromeric index and number of supernumerary chromosomes (Ribas, 1990). Inclusion of representatives of this species in molecular phylogenetic studies revealed very high genetic divergences between them and, in some cases, the species was not recovered as a monophyletic unit (Baguñà et al., 1999; Lázaro et al., 2009). Furthermore, a recent study on the impact of reproductive strategies on the genetic characteristics of individuals of this species showed that many individuals present very high levels of mosaicism and intraindividual genetic diversity due to their fissiparous reproduction (Leria et al., 2019). 
In view of these previous studies on possible differentiation within the current nominal species D. subtentaculata (further below referred to as $D$. subtentaculata sensu lato (s.l.)), we considered it opportune to conduct a systematic revision of this species, not only for finally establishing its taxonomic status but also for exploring how inclusion of karyological data and up-to-date molecular methodologies might improve the usually complex species delimitation procedures in the genus Dugesia in general. Additionally, the existence of mosaicism and high intraindividual genetic diversity in this species makes the application of these new methodologies challenging and, therefore, D. subtentaculata forms a good model species to test whether these methods form a reliable tool for molecular species delimitation in planarians as well as other organisms with similar genetic characteristics. In view of our aim to perform an integrative species delimitation analysis of $D$. subtentaculata s.l., our procedure consisted of the following four sequential steps: (1) an extensive sampling across the species' distributional range, (2) a search for new, phylogenetically informative nuclear markers by means of a low-coverage genome sequencing approach, (3) formulation of PSHs on the basis of three different molecular discovery methods applied to a single mitochondrial locus and a single nuclear locus, with and without intraindividual information, and (4) formulation of SSHs by validating the PSHs with multilocus data (using a molecular validation method), morphological data, and karyological data.

\section{Materials and methods}

\subsection{Taxon sampling}

We sampled a total of 200 localities across all fluvial basins of the Iberian Peninsula and Southern France, including the species' type locality at Montpellier (France), as well as the two known populations from Mallorca (Balearic Islands) previously assigned to D. iberica. Moreover, Dugesia individuals from about 20 additional localities in the Iberian Peninsula and Northern Africa were obtained from collaborators. Thus, our dataset not only covered almost the complete distributional range of the species but even extended it considerably. Dugesia subtentaculata s.l. was found at 63 of these localities, which were all used in the present study (Fig. 1, Supplementary data S1). From each population some of the animals that showed a copulatory apparatus (indicated by presence of a gonopore at the ventral surface of the animal) were cut into two pieces: the anterior part of the individual (from the head to the prepharyngeal region) was fixed and stored in $100 \%$ ethanol for subsequent molecular work, while the rest of the body was fixed in Steinmann's fluid (see Winsor and Sluys, 2018) and thereafter preserved in $70 \%$ ethanol for morphological analysis of the copulatory apparatus. However, most of the animals from each of the populations that showed a copulatory apparatus were fixed entirely in Steinmann's fluid in order to have anatomical information for the whole body. Animals devoid of a copulatory apparatus were fixed in $100 \%$ ethanol. Additionally, some animals from each population were kept alive for karyological analysis. A few samples made available to us by colleagues (some populations from the Iberian Peninsula and Northern Africa) were used only for molecular analyses, as all of these animals were fixed in $100 \%$ ethanol and, therefore, were less suitable for histological studies.

\subsection{Search for new molecular markers}

\subsubsection{Selection of individuals to be sequenced at genomic level}

In order to identify new nuclear genes with adequate levels of variability within the species, we sequenced at low-coverage the genome of individuals from different populations, which in a Cox1based phylogenetic tree showed among them different levels of genetic divergence. For constructing that tree, we extracted genomic DNA from at least two specimens from each population sampled, using the commercial reagent DNAzol (Molecular Research Center Inc., Cincinnati,
$\mathrm{OH}$ ), following the manufacturer's instructions. Subsequently, the mitochondrial gene Cox1 was PCR-amplified for all individuals, using the primers and PCR conditions described in Solà et al. (2013). The phylogeny was inferred through Bayesian Inference using the program MrBayes 3.2 (Ronquist et al., 2012), running 1,500,000 generations and sampling a tree every 150 generations. Phylogenetic inference was carried out with two independent runs and with a burn-in of $25 \%$ to infer the tree topology and the posterior probability of the nodes. Before applying the burn-in, it was checked through the standard deviation of splits value that convergence of the two runs had been achieved and that each run had arrived at the stationary region. The substitution model was previously determined with jModelTest2 (Darriba et al., 2012). Subsequently, using the Cox 1 phylogeny as a reference, we chose four individuals belonging to the populations 15, 22, 46 and 48 for sequencing their genome at low-coverage. Additionally, we selected also some individuals from a population of Dugesia sicula Lepori, 1948 from Mallorca to be sequenced at the genome level, as this species is genetically highly differentiated from $D$. subtentaculata s.l. (Lázaro et al., 2009) and thus would facilitate detection of highly conserved regions for primer design.

\subsubsection{High-quality DNA extraction and genome sequencing}

We first estimated the necessary sequencing effort for reaching a $4 \mathrm{x}$ coverage per genome, which would be sufficient for discovering new molecular markers for our study. Therefore, by flux cytometry (protocol in Supplementary data S2) we inferred the size of the haploid genome of selected populations of $D$. subtentaculata s.l. and D. sicula. Thereafter, $1 \mu \mathrm{g}$ of high-quality DNA was extracted from single individuals from each of the four populations of D. subtentaculata s.l., using a customized phenol-chloroform protocol; for D. sicula DNA extraction was performed on a pool of three individuals. Tissue was digested overnight at $37^{\circ} \mathrm{C}$ in a solution containing $200 \mu \mathrm{L}$ of Lysis Buffer Solution (Wizard ${ }^{\circledR}$, Promega, Madison, WI, USA) and $10 \mu \mathrm{L}$ of Proteinase $\mathrm{K}(20 \mathrm{mg} / \mathrm{mL})$. Then $12 \mu \mathrm{L}$ of RNase A $(10 \mathrm{mg} / \mathrm{mL})$ was added and the solution was incubated during $1 \mathrm{~h}$ at $37^{\circ} \mathrm{C}$. Finally, the standard phenol-chloroform extraction protocol of Sambrook et al. (1989) was followed. Quality and quantity of total DNA was examined using a Qubit 2.0 Fluorometer (Invitrogen). The whole genome of these individuals was sequenced in a lane of the Illumina Hi-Seq2000 (tagged paired-end libraries) in Macrogen Inc., South Korea (www.macrogen.com). Raw data of each genome was filtered for poor quality and low complexity reads by using the subprogram "preprocess" from the SGA pipeline (with "- dust" and "-quality-filter $=30$ " options). In order to generate contigs that we could later blast against Schmidtea mediterranea's (Benazzi, Baguñà, Ballester, Puccinelli, \& Del Papa, 1975) genome, we mapped these preprocessed genomic reads onto the reference transcriptome assembly of Dugesia japonica Ichikawa \& Kawakatsu, 1964 (Chan et al., 2016) using the faster mapping option based on BWA (Li and Durbin, 2009) of Stampy (Lunter and Goodson, 2011).

\subsubsection{Marker selection}

Dugesia contigs were blasted against the genome of Schmidtea mediterranea SXl v4.0, available online at SmedGD (http://smedgd.stowers. org) (Robb et al., 2015), and contigs corresponding to single copy genes containing introns flanked by conserved exonic regions were selected. Primers were designed for a total of 23 markers and were PCR-tested by using one individual from each Cox1 main clade. Finally, six markers that showed a mean divergence between the different populations ranging from $2 \%$ to $6 \%$ were used for the present study. These markers, which were named Dunuc's (from Dugesia nuclear) followed by a number, corresponded to: (1) a MAP Kinase death domain (Dunuc2), (2) an anonymous marker (Dunuc3), (3) a disulphide isomerase (Dunuc5), (4) a translation initiation factor (Dunuc10), (5) a transport protein transmembrane domain (Dunuc12, referred to as TMED9 in Leria et al., 2019), and (6) a transcription factor (Dunuc20). 


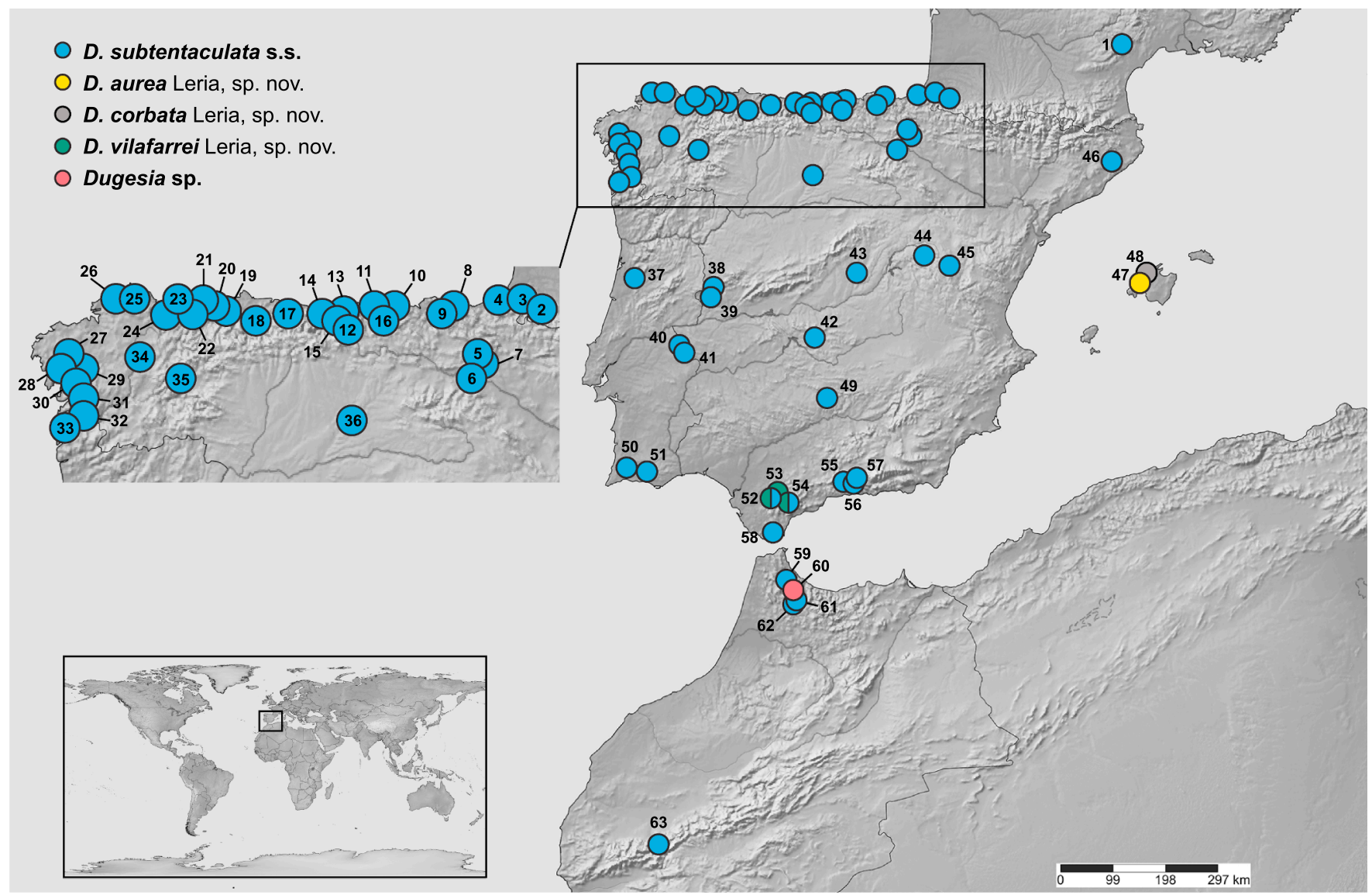

Fig. 1. Geographic populations of the Dugesia subtententaculata s.l. species complex examined in the present study. Numbers correspond to population codes listed in Supplementary data S1. At localities 52 and 54 D. vilafarrei and D. subtentaculata s.s., occur together, as indicated by the two separate halves of the circles filled with their respective colours.

\subsection{DNA sequences and datasets}

In addition to the two individuals per locality that were PCR-amplified for Cox1, nine additional gene fragments were amplified for a subset of individuals belonging to the 20 main Cox1 clades. These gene fragments included: (a) six new nuclear fragments (Dunuc2, Dunuc3, Dunuc5, Dunuc10, Dunuc12 and Dunuc20), (b) $28 S$ ribosomal 1 (28S), (c) Internal Transcribed Spacer 1 (ITS), (d) a mitochondrial fragment containing part of the NADH: ubiquinone oxidoreductase core subunit 1 (Nad1), (e) transfer RNA-Trp (tRNA-W), and (f) part of Cytochrome c oxidase II (Cox2). Amplification of the two ribosomal genes was carried out by using already available primers (Álvarez-Presas et al., 2008; Baguñà et al., 1999), while the mitochondrial fragment was amplified with the help of newly designed primers by using the available mitogenomes of D. japonica and D. ryukyuensis Kawakatsu, Oki, Tamura \& Sugino, 1976 as reference (Sakai and Sakaizumi, 2012). The Cox1 and Dunuc12 gene fragments were also PCR-amplified for several individuals of different Dugesia species closely related to $D$. subtentaculata s.l. (Lázaro et al., 2009; Solà, 2014), which were used as outgroup taxa in the various molecular species delimitation analyses, viz. D. hepta Pala, Casu \& Vacca, 1981, D. benazzii Lepori, 1951, D. etrusca Benazzi, 1944, and D. liguriensis De Vries, 1988. Primer sequences and PCR conditions used in this study are detailed in Supplementary data S3.

Amplification products were purified using a vacuum system (MultiScreen ${ }^{\text {TM }}$ HTS Vacuum Manifold of Millipore) and were subsequently sequenced in both directions at Macrogen Europe, Inc. (Amsterdam). Complementary strands of DNA were assembled into consensus using Geneious R8 (Biomatters, http://www.geneious.com/). All sequences were deposited in GenBank (GenBank accession numbers detailed in Appendix A).

Finally, we took profit of sequences obtained in a parallel study on the intraindividual genetic variability of this species, for which we obtained intraindividual sequences by cloning the PCR products of the Cox1 and Dunuc12 genes for each individual (Leria et al., 2019). In the present study, we have included all intraindividual sequences (on average 10 per individual) of the two genes for 32 individuals (belonging to the main Cox1 clades). GenBank accession numbers of sequences used are MK385658 to MK385866 (Dunuc12) and MK385871 to MK385922 (Cox1).

Sequences of all gene fragments were separately aligned using the online software MAFFT (version 7) (Katoh and Standley, 2013) and revised with Geneious R8. The protein coding genes were translated into amino acids to check the reading frame (genetic code 9 was used for the mitochondrial genes, while genetic code 1 was used for the nuclear genes). Degree of sequence saturation of all alignments was checked with the program DAMBE (Xia and Xie, 2001) by performing a substitution saturation test (Xia et al., 2003; Xia and Lemey, 2009). The non-coding genes and the non-coding regions (introns) were analyzed at the nucleotide level, while the protein coding genes and the protein coding regions (exons) were analyzed at the three codon positions.

Aligned sequences of the 12 loci were organized into 7 different datasets to be used in the various molecular species delimitation analyses: (1) Cox1, (2) Dunuc12, (3) Cox1-Cloned (intraindividual Cox1 sequences), (4) Dunuc 12-Cloned (intraindividual Dunuc12 sequences), (5) All (12 loci), (6) Nuclear (6 Dunuc loci) and (7) Mitochondrial (4 mitochondrial loci) (Appendix A). In the datasets with no intraindividual information, each individual was represented by a single sequence (in some cases with polymorphic sites), while in the Cloned 
datasets the 32 selected individuals had many sequences without polymorphic sites, corresponding to their different intraindividual haplotypes.

\subsection{Integrative taxonomic procedure}

Our integrative taxonomic procedure required the formulation of initial Primary Species Hypotheses (PSHs). These PSHs were based on the most often recurring partition of species obtained, after independent application of three different molecular discovery methods of species delimitation to the datasets 1, 2, 3 and 4 (single locus datasets with or without intraindividual information). The methods used in this step were: (a) Automatic Barcode Gap Discovery (ABGD; Puillandre et al., 2012), (b) multi-rate Poisson Tree Processes (mPTP; Kapli et al., 2017), and (c) General Mixed Yule-Coalescent (GMYC; Pons et al., 2006). The PSHs were subsequently validated, or not, by different lines of evidence: (a) multilocus data (datasets 5, 6 and 7) by using the coalescent-based method incorporated in the software Bayesian Phylogenetics and Phylogeography (BPP; Yang and Rannala, 2010); (b) morphological data; (c) karyological data. The results of these three sources of information were integrated in order to generate the Secondary Species Hypotheses (SSHs), eventually leading to pertinent taxonomic decisions (Fig. 2).

\subsection{Molecular methods for species delimitation}

\subsubsection{Discovery}

ABGD is a distance-based method that uses a DNA alignment to determine the threshold between intraspecific and interspecific diversity (the barcode gap). Genetic distances of each alignment were calculated with the help of the program MEGA version 5 (Tamura et al., 2011) under the Kimura-2-parameters model, while the resulting distance matrix was imported into the ABGD web-interface (available at http://wwwabi.snv.jussieu.fr/public/abgd/abgdweb.html). All parameters were left as default, excepting the relative gap width, which was set to 1 , since we are working on closely related candidate species. In each analysis the selected partition scheme corresponded to the maximum value of intraspecific genetic diversity $(P)$ that delimited the outgroups as different species.

The MPTP method uses the number of substitutions along the branches of a phylogenetic tree to determine putative species, based on the assumption that intraspecific and interspecific substitutions follow distinct Poisson distributions. Moreover, this method allows different substitution rates to take place at intraspecies level. Before estimating the phylogenetic trees, we determined the best substitution model for each dataset, using jModelTest2. In both Cox1 datasets the molecular evolutionary model determined was the HKY + Gamma + Invariant sites, while for the Dunuc12 it was GTR + Gamma. The input phylogenies were obtained by using Maximum Likelihood with the program RaxML 7.0.0 (Stamatakis, 2006), with 2000 replicates to obtain bootstrap support. For the Cox1 datasets we also used the model GTR, since that model is the only one implemented in the program RaxML. To run the mPTP analyses we used the command line version (Kapli et al., 2017). All analyses were conducted with 4 independent runs of 5,000,0000 Markov chain Monte Carlo (MCMC) generations, sampling at every 10,000 generations in order to obtain the support values for each delimitation scheme. As all identical sequences were removed prior to the phylogenetic inference, no minimum branch length (minbr) was used.

GMYC identifies the transition between intra- and interspecific branching rates in an ultrametric tree by modelling speciation via a pure birth process (Yule model) and intraspecies divergence via coalescence. The ultrametric tree for each dataset was inferred by using the software BEAST v.1.7.4 (Drummond et al., 2012). For the Cox1 datasets we set as site priors: substitution model $=\mathrm{HKY}$; bases frequencies = empirical; site heterogeneity model = gamma + invariant sites; number of gamma categories $=4$. For the Dunuc12 datasets we set as site priors: substitution model $=\mathrm{GTR}$; bases frequencies $=\mathrm{em}$ pirical; site heterogeneity model = gamma; number of gamma categories $=4$. For the Cox1, a lognormal relaxed molecular clock with a mean value of 0.017 substitutions per site per million years was used for time-calibrating the tree, a mean value that was estimated for the genus Dugesia (Solà et al., 2013), while for the nuclear marker the rate parameters were left as default. For both molecular markers the Yule Process was used as speciation model (Gernhard et al., 2008). For the datasets that contained polymorphic sites we set BEAST to use the information of the ambiguous codes, since by default the polymorphic sites in BEAST are treated as missing data. Equal sequences were removed prior to the analyses. Runs were conducted in CIPRES Science Gateway (Miller et al., 2010) with 50,000,000 generations and sampling every 5,000 generations. The resulting log files were examined in Tracer 1.7 (Rambaut et al., 2018) to check that the Effective Sample Size (ESS) values for the different parameters were higher than 200. TreeAnotator (available in the BEAST package) was used to construct the ultrametric trees, using a burn-in of $10 \%$. The ultrametric trees obtained with BEAST were submitted to SPLITS package for R (SPecies LImits by Threshold Statistics; Ezard et al., 2009; available at http://rforge.r-project.org/projects/splits/), which implements GMYC. The analyses were conducted under the single-threshold approach, while always checking that the results of the tests were significant.

\subsubsection{Validation}

BPP can use the information of multiple genes under the multispecies coalescent model (Rannala and Yang, 2003) to evaluate

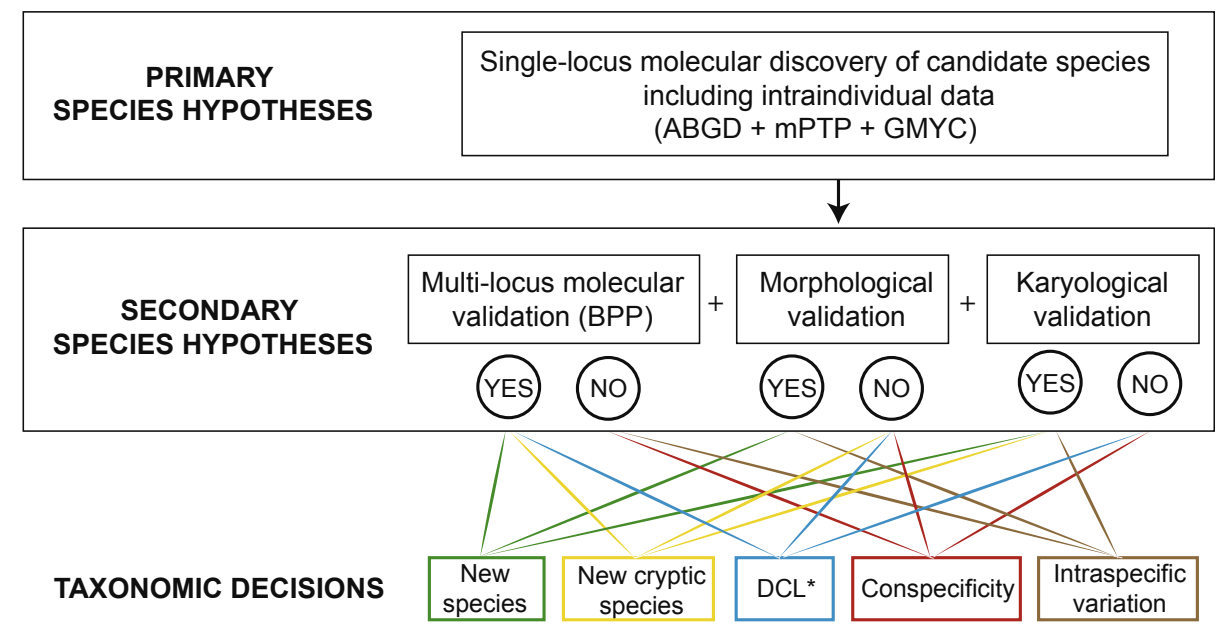

Fig. 2. Integrative taxonomic procedure used in this study. Formulation of Primary Species Hypotheses (PSHs) was based on the most recurrent partition obtained after independent application of three methods of molecular species delimitation (ABGD, mPTP, and GMYC) to four single-locus datasets (nuclear, mitochondrial, nuclear with intraindividual information, and mitochondrial with intraindividual information). Secondary Species Hypotheses (SSHs) were obtained through validation of PSHs based on multi-locus molecular data (applying BPP), morphological data, and karyological data, leading to pertinent taxonomic decisions. *: Deep Conspecific Lineage. 
whether the different nodes of a given phylogeny should be collapsed or retained, depending upon whether coalescent or speciation processes are adequate explanations (model A10, species delimitation from a fixed guide tree) (Yang, 2015). The topology of the guide tree for the BPP analyses was inferred with *BEAST (Heled and Drummond, 2010) using dataset 5 (all loci) with the following priors, independently calculated for each locus: substitution model $=\mathrm{HKY}$; bases frequencies = empirical; site heterogeneity model = gamma; number of gamma categories $=4$; clock type: uncorrelated lognormal relaxed; Species tree = Yule Process; Population Size Model: Piecewise linear \& constant root. The analysis was run in Portal CIPRES, setting 100 million generations and sampling every 10,000. Convergence was assessed in Tracer by checking the ESS values. The species tree was estimated by using $D$. hepta and $D$. benazzii as outgroups. However, the BPP analyses were conducted by using the sequences of $D$. subtentaculata s.l. alone.

As BPP needs prior information on ancestral population size $(\theta)$ as well as divergence time from the root $(\tau)$, we tested four possible different scenarios for our PSHs in three different BPP analyses, using the multilocus datasets 5, 6 and 7: (1) M1: small ancestral population size and shallow divergence (G (2 1000) for $\theta$ and G (2 1000) for $\tau$ ); (2) M2: large ancestral population size and shallow divergence (G (1 10) for $\theta$ and G (2 1000) for $\tau$ ); (3) M3: large ancestral population size and deep divergence (G (1 10) for $\theta$ and $\tau$ ); (4) M4: small ancestral population size and deep divergence (G (2 1000) for $\theta$ and G (1 10) for $\tau$ ). The combination of these priors with the three datasets gave a total of 12 different partitions to be tested with BPP.

\subsection{Morphological data}

Specimens that had been preserved in Steinmann's fluid were cleared in clove oil and subsequently embedded in synthetic wax. Sagittal sections were made at intervals of $8 \mu \mathrm{m}$ and horizontal sections at intervals of $7 \mu \mathrm{m}$ and, subsequently, were stained in Mallory-Cason/ Heidenhain (cf. Winsor and Sluys, 2018) and mounted in DPX. Reconstructions of the copulatory apparatus were obtained by using a camera lucida attached to a compound microscope. In order to clearly visualize some anatomical structures, we also made a three-dimensional (3D) reconstruction of the copulatory complex from digitized images of serial histological sections, using the software Free-D (Andrey and Maurin, 2005). All specimens used for the morphological analysis were deposited in the collections of the Naturalis Biodiversity Center, Leiden, The Netherlands (Supplementary data S4).

Besides performing a morphological study of the different PSHs recognized within $D$. subtentaculata s.l., we also compared the morphological characteristics of the new material with the histological sections of the neotype of this species (deposited in the collections of Naturalis Biodiversity Center).

\subsection{Karyological data}

Individuals selected for the karyological analysis were cut into two pieces and left to regenerate for 4 days in a 1:1 mixture of tap water and distilled water at $20^{\circ} \mathrm{C}$. Then, the specimens were incubated in a solution of $0.075 \%$ colchicine for $6 \mathrm{~h}$. Next, we washed the animals with a solution of $0.5 \% \mathrm{~N}$-acetyl-L-cysteine for $1 \mathrm{~min}$ before fixing them in a freshly prepared mixture of methanol:glacial acetic acid (3:1) and, subsequently, incubating them for $20 \mathrm{~min}$ in $40 \%-45 \%$ glacial acetic acid. After incubation, each animal was placed onto a glass slide, and the region of the blastema and post-blastema was minced with a surgical blade and suspended in $20 \mu \mathrm{L}$ of glacial acetic acid. The macerated cell suspension was dropped onto preheated glass slides (at $65^{\circ} \mathrm{C}$ ) and were left to air-dry. Thereafter, chromosome preparations were stained with 1:20 Giemsa:tap water mixture for one minute, dried, and then sealed under a cover slip by using DPX. All karyological preparations were deposited in Dpt. de Genètica, Microbiologia i Estadística; Facultat de Biologia; Universitat de Barcelona, Barcelona, Spain (Supplementary table S5).

An average of 5 metaphasic plates per individual were photographed and subsequently analyzed with the program Karyotype (Altinordu et al., 2016) in order to determine ploidy level, centromeric indices, and relative lengths of the chromosomes. Classification of the chromosomes on the basis of their centromeric index followed Levan et al. (1964). Chromosome measures of D. subtentaculata populations published by De Vries (1986b) and Ribas (1990) were included in the analysis.

Abbreviations used in the figures: bc, bursal canal; ca, common atrium; cb, copulatory bursa; cg, cement glands; dp, diaphragm; ed, ejaculatory duct; ec, ectal reinforcement; go, gonopore; mg, musculoglandular structure; od, oviduct; pb, penis bulb; pp, penis papilla; sg, shell glands; sv, seminal vesicle; vd, vas deferens.

\section{Results}

\subsection{Geographic distribution of D. subtentaculata s.l.}

The extensive samplings carried out for this study increased the number of known localities of D. subtentaculata s.l. from 13 to 67 . Interestingly, $D$. subtentaculata s.l. reaches its maximum abundance in the northern sector of the Iberian Peninsula, an area where the species was not detected previous to our study. In contrast, its occurrence in southern France is restricted to the type locality (near Montpellier) and two other localities at the western coast near the Basque country (Fig. 1; Supplementary data S1). The localities where we did not find $D$. subtentaculata s.l. were either occupied by other Dugesia species, other planarian species, or did not have any freshwater triclad fauna at all (Supplementary data S6).

\subsection{Low coverage genome assembly}

We estimated a haploid genome size of approximately $2 \mathrm{~Gb}$ for both $D$. subtentaculata s.l. and D. sicula. The average of total bases sequenced per species was $7.85 \mathrm{~Gb}$ and $7.1 \mathrm{~Gb}$, which corresponded to a coverage across the genome of $3.93 \mathrm{X}$ and $3.55 \mathrm{X}$, for D. subtentaculata s.l. and D. sicula populations, respectively (with a very slight variation in the coverage of each individual population). After the read pre-processing, the number of reads used for the mapping step varied between $85 \%$ and $90 \%$ of raw reads, depending on the population. However, the final percentage of these pre-processed genomic reads aligned with $D$. japonica transcripts was only a $5.7 \%-7.3 \%$, since the rest of the reads corresponded to freshwater protozoans. The number of $D$. japonica transcripts mapped with reads of the other species ranged from 21,087 ( $D$. subtentaculata s.l. population 22) to 28,612 (D. sicula), although the proportion of the transcripts covered by genomic reads was highly variable. The consensus sequences across the populations of $D$. subtentaculata s.l. and D. sicula for these mapped regions were used as queries in BLAST searches for marker discovery (see methods).

\subsection{Molecular datasets}

A total of 840 new sequences of $D$. subtentaculata s.l. were obtained for the present study (Appendix A), representing approximately 7300 aligned characters. Information on each dataset, including number of sequences, alignment length, and number of variable sites, is detailed in Supplementary data S7. No stop codons were detected in the protein coding genes. Further, no gene, gene fragment, or codon position showed significant levels of sequence saturation, as in all cases the Index of Substitution Saturation was significantly lower than the Critical Index of Substitution Saturation (Supplementary data S8).

\subsection{Single-locus discovery of candidate species}

Phylogenetic inferences based on the Cox1 and Dunuc12 datasets 


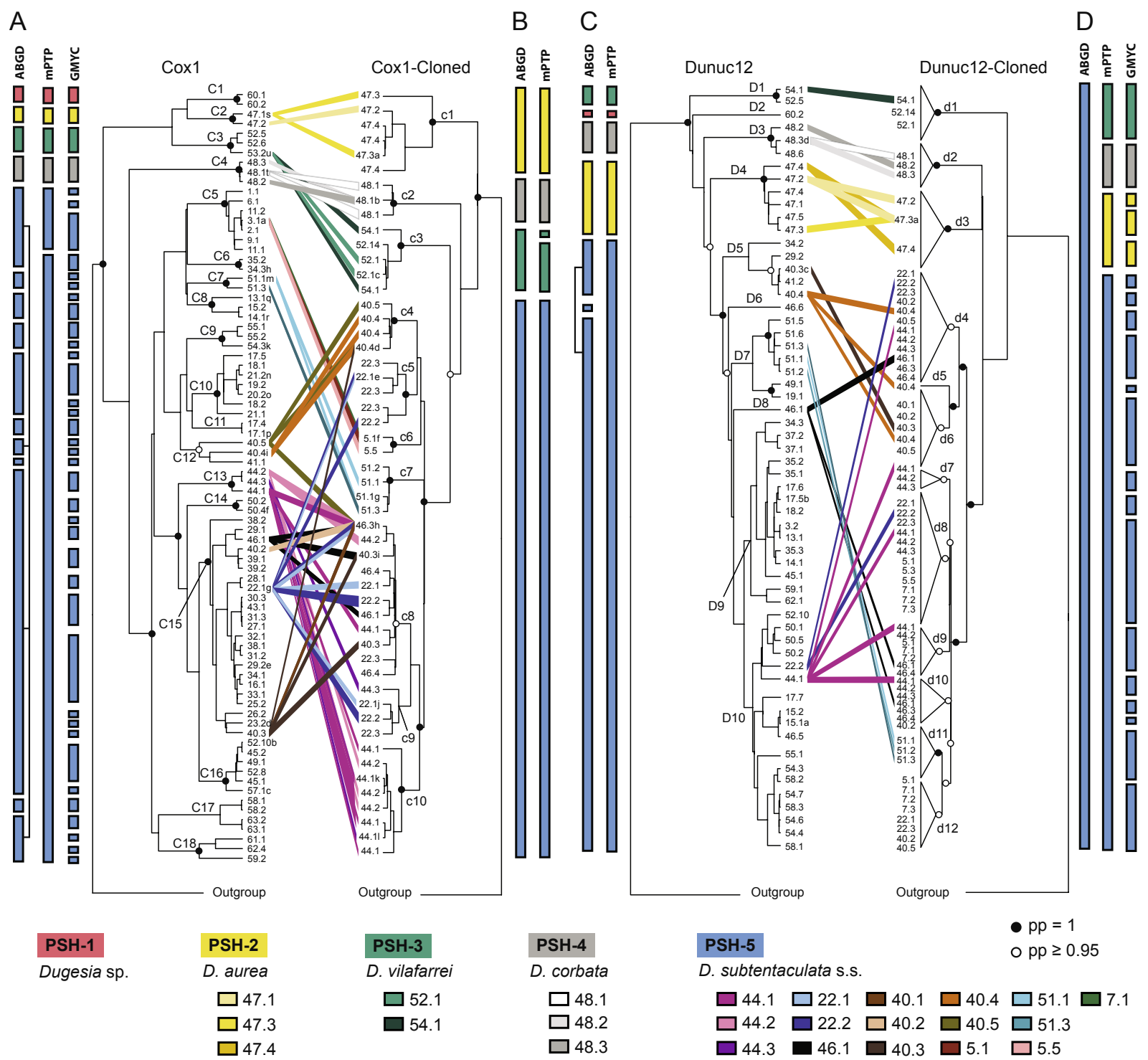

Fig. 3. Summary of the results obtained for the three molecular species delimitation methods (ABGD, mPTP and GMYC) applied to the Cox1 dataset (A), Cox1-Cloned dataset (B), Dunuc12 dataset (C), and Dunuc12-Cloned dataset (D). The partition scheme obtained for each method/dataset is indicated by coloured boxes next to the ultrametric Bayesian phylogenetic tree inferred with BEAST; boxes connected by thin black lines belong to the same candidate species. Colours of boxes correspond to the Primary Species Hypotheses resulting from the analysis. Coloured lines connect the haplotypes of some individuals (see graphical legend) obtained by direct PCR with the haplotypes of the same individuals obtained by cloning. Letters (a to v) after the codes of the individuals denote that the same haplotype was found in other individuals (Supplementary data S9). Nodes without circles indicate a pp $<0.95$. See Supplementary data S10 for the non-collapsed Dunuc12-Cloned tree.

without intraindividual information resulted in similar topologies (Fig. 3A and Fig. 3C, respectively). In both phylogenies the first diverging populations were the following: $47,48,60$, and some individuals of populations 52-54. Individuals of these populations were structured in four different clades, clades C1 to C4 and D1 to D4 in Fig. 3A and Fig. 3C, respectively (further below the individuals from localities 52-54 that conform the clades C3 and D1 will be referred as 52-54*). Although each of these four clades was highly supported, evolutionary relationships between them were not fully resolved in any of the two phylogenies. The remaining populations were structured in 13 main clades in the Cox1 phylogeny (from clade C5 to clade C18 in Fig. 3A) and in 6 main clades in the Dunuc 12 phylogeny (from clade D5 to clade D10 in Fig. 3C). Additional phylogenetic inferences of both genes, now using intraindividual information of individuals belonging to different clades (Fig. 3B, D), recovered each of the clades formed by the populations 47,48 , and $52-54 *$ as monophyletic (no cloned information on population 60 being available) (clades $\mathrm{c} 1$ to $\mathrm{c} 3$ and $\mathrm{d} 1$ to d3 in Fig. 3B and Fig. 3D, respectively). The haplotypes of the rest of individuals were structured in 7 different clades in the Cox1-Cloned dataset (which did not have an exact match with the clades of the Cox1 dataset) and in 6 different clades in the Dunuc12-Cloned dataset (again with different correspondence with the Dunuc12 dataset clades) (Fig. 3B, D). Lack of correspondence between the clades of the cloned and non-cloned datasets was due, on the one hand, to the fact that on the basis of the cloned datasets, haplotypes of the same individual were distributed in different clades (e.g., individual 22.2 in Fig. 3B, D). On the other hand, it turned out that individuals that occurred in different clades in the non-cloned datasets, shared some haplotypes or showed very similar haplotypes in the cloned datasets (e.g., individuals 40.5C12 and 44.2-C13 share a haplotype named 44.3 h-c8; Fig. 3A, B).

\subsection{1. $A B G D$}

The ABGD method applied to the Cox1 dataset delimited 12 different groups within D. subtentaculata s.l. (P 0.0027-0.0077) (Fig. 3A). But when this method was applied on the Cox1-Cloned dataset it retrieved only four groups within $D$. subtentaculata s.l. $(\mathrm{P}=0.0077)$ 


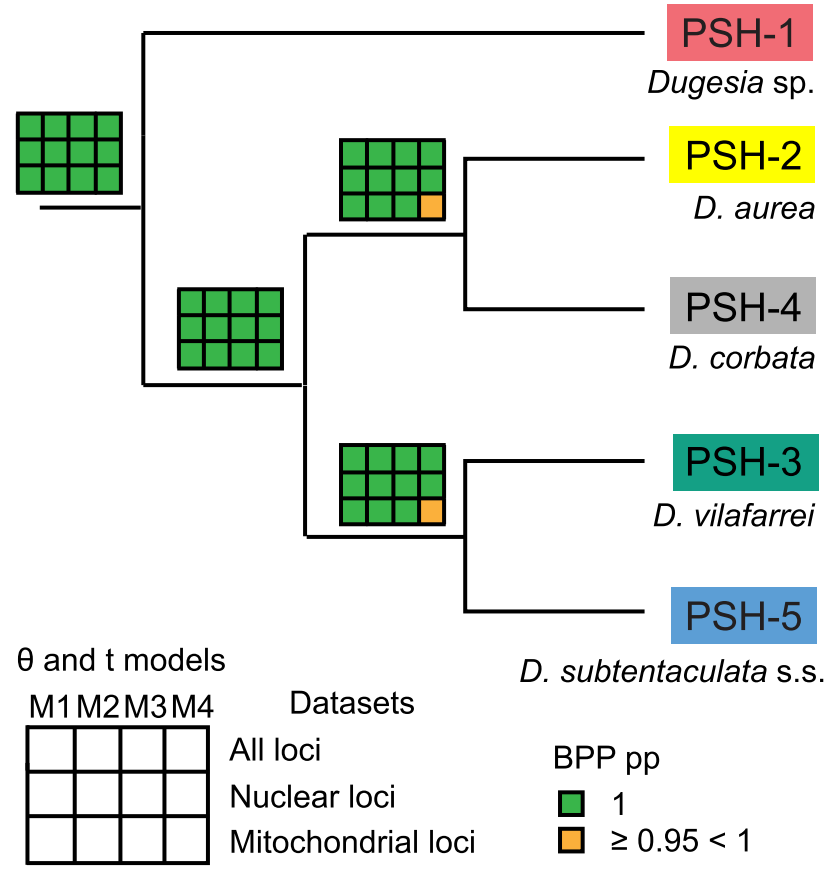

Fig. 4. Schematic representation of the results obtained with BPP on multilocus data. Colours of squares indicate the posterior probability of each node for each of the 12 BPP analyses. The topology was obtained with *BEAST for the multilocus dataset containing all loci (Supplementary data S11). Colour codes of the PSHs are the same as used in Fig. 3.

(Fig. 3B). Three of these groups were the same for both datasets, corresponding to the populations 47,48 , and $52-54 *$. The fourth candidate species for the Cox1-Cloned dataset comprised the rest of the populations, excepting number 60 , which was not represented in the cloned dataset (this population was retrieved as an independent group in the dataset with no intraindividual information).

The ABGD method applied to the nuclear marker Dunuc12 recognized six different groups within D. subtentaculata s.l. (P 0.0028-0.001) (Fig. 3C). Four of these groups coincided with the Cox1 results by delimiting the following populations as candidate species: 47 , $48,52-54^{*}$, and 60 . One of the other two groups was formed by a singleton from population 46 , while the other group comprised the rest of the populations. ABGD on the Dunuc12-Cloned dataset retrieved all D. subtentaculata s.l. populations as a single species (P 0.0028-0.001) (Fig. 3D).

\subsection{2. $M P T P$}

The mPTP method delimited for the Cox 1 dataset six candidate species within the group of $D$. subtentaculata s.l. populations, with an average support of 0.93 (Fig. 3A). Four of these candidate species corresponded to groups already delimited by the ABGD analysis of this dataset, viz. populations $47,48,52-54 \%$, and 60 (all with a posterior probability (pp) of 1); the rest of the populations was divided into two groups ( $\mathrm{pp}=0.8$ ). In the Cox1-Cloned dataset $(\mathrm{pp}=0.93$ ) populations 47,48 , and 52-54* were also retrieved as different candidate species (pp of 1,1 and 0.72 , respectively), while the rest of the populations formed a single group ( $p p=1$ ) (Fig. 3B). Surprisingly, in the Cox1Cloned dataset one haplotype of individual 54.1 was positioned as representing a single candidate species $(\mathrm{pp}=0.72)$.

In the case of the nuclear Dunuc12 and Dunuc12-Cloned datasets, the MPTP method delimited for both datasets the same four groups within the D. subtentaculata s.l. populations (with an average pp of 0.93 and 0.82 , respectively), viz. populations $47,48,52-54 *$, and all the remaining ones (Fig. 3C, D). In addition, population 60 was also delimited as a species in the Dunuc12 dataset (this population is not present in the Dunuc12-Cloned dataset). The pp values for all groups were higher than 0.8 in both datasets, excepting the group formed by individuals of population 47 and the group comprising the rest of the populations on the Dunuc12 dataset ( $\mathrm{pp}=0.77$ ).

\subsection{3. $G M Y C$}

The GMYC analysis of the Cox 1 dataset delimited populations 47 , $48,52-54 *$, and 60 as separate candidate species, while the rest of the populations were spread over no less than 36 different groups (Fig. 3A). However, support values for all these 36 groups were very low. In contrast, the Cox1-Cloned dataset produced no significant differences between the likelihood of the GMYC model and the null model (GMYC model $=153.5169$, null model $=152.7937$, likelihood ratio $=1.44$ and result of LR test: 0.485 n.s.), so that no candidate species could be delineated on the basis of this dataset.

The same result was obtained for the Dunuc12 dataset (GMYC model $=258.54$, null model $=257.91$, likelihood ratio $=1.24$ and result of LR test: 0.53 n.s.). However, the GMYC analysis performed on the Dunuc12-Cloned dataset did give significant results, in that populations $52-54^{*}$ and 48 (clades $\mathrm{d} 1$ and $\mathrm{d} 2$, respectively) were retrieved as different candidate species, with high support values, while the remaining populations were arranged into 18 different groups, albeit with low support values (Fig. 3D). In the last-mentioned case, haplotypes of the same individual were distributed over two, three, four, or even five different candidate species.

\subsection{Primary Species Hypotheses}

Our analysis using three molecular methods for species delimitation most frequently identified the following populations as candidate species: $47,48,52-54^{*}$, and 60 (although population 60 was only available for the datasets without cloned information). The cloned datasets indicated that the remaining populations all constituted a single candidate species (excepting on the GMYC analysis), while in most cases the datasets without intraindividual information arranged these populations into different candidate species, because of their inability to detect that the genetic diversity occurred at the intraindividual level. Therefore, our Primary Species Hypotheses were: PSH-1 (population 60), PSH-2 (population 47), PSH-3 (populations 52-54*), PSH-4 (population 48), and PSH-5 (populations 1-63, excepting the ones constituting the other PSHs).

\subsection{Validation of the Primary Species Hypotheses}

\subsubsection{Multilocus molecular validation}

The species tree obtained with *BEAST for the multilocus dataset (Supplementary data S11) resulted in the following topology: PSH-1 was sister to a group including the other four PSHs, which comprised two sister-groups, one formed by PSH-2 and PSH-4 (two lineages from Mallorca) and the other by PSH-3 and PSH-5. The results of the BPP analyses for the three multilocus datasets using this topology (Fig. 4) suggested that the genetic differentiation among these five PSHs might be explained by speciation rather than by coalescent processes, as in all cases all nodes were recovered with a high posterior probability. Different prior values of $\theta$ (ancestral population size) and $\tau$ (divergence time) did not have a significant effect on the results of the BPP analyses in any of the datasets, excepting the mitochondrial dataset, in which the pp for the nodes PSH-2/PSH-4 and PSH-3/PSH-5 changed from 1 to 0.99 in the M4 model (small ancestral population size and deep divergence). Therefore, the multilocus molecular test validated our 
A

$\mathrm{PSH}-2$

$\mathrm{PSH}-3$

D. aurea

D. vilafarrei
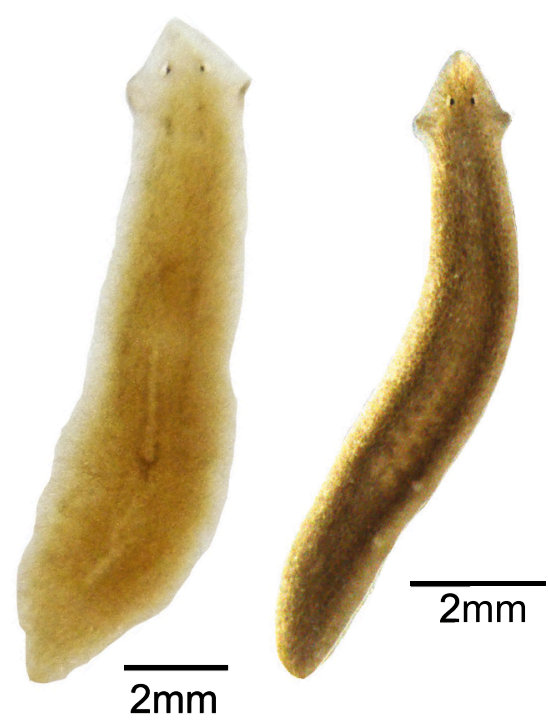

B

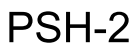

D. aurea

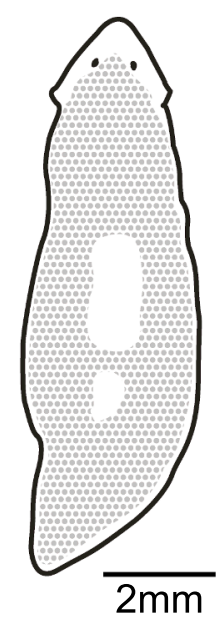

$\mathrm{PSH}-3$

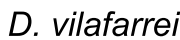

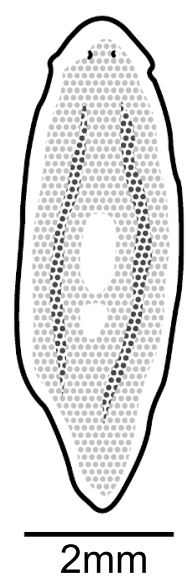

$\mathrm{PSH}-4$

D. corbata

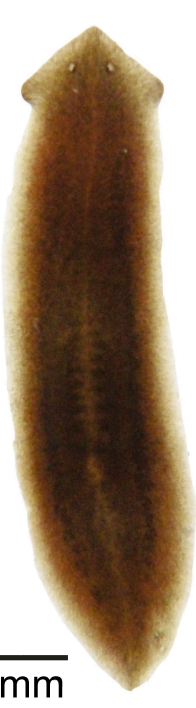

$\mathrm{PSH}-5$

D. subtentaculata s.s.

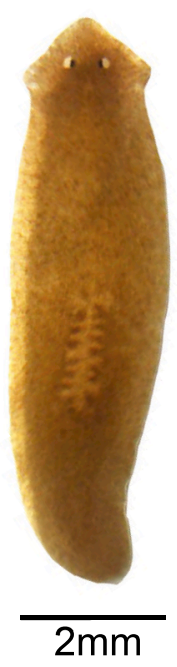

$\mathrm{PSH}-4$

D. corbata

$\mathrm{PSH}-5$

D. subtentaculata s.s.

Fig 5. External morphology of the different PSHs.
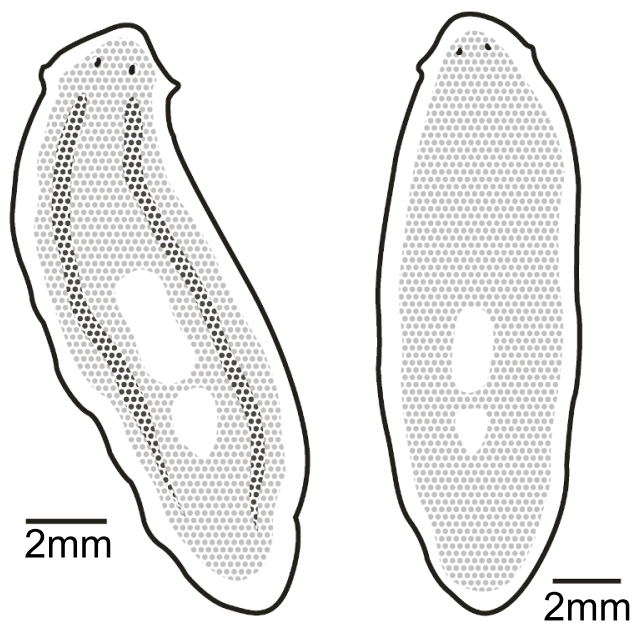

. (A) Dorsal photographs of live specimens. (B) Drawings of specimens preserved in clove oil in dorsal view.

Primary Species Hypotheses.

\subsubsection{Morphological validation}

In our search for morphological diagnostic characters, we analyzed external characteristics as well as the anatomy of the copulatory apparatus of 4 individuals of PSH-2, 2 individuals of PSH-3, 3 individuals of PSH-4 and 29 individuals from 12 different populations of PSH-5 (Supplementary data S4). It was not possible to analyze individuals of PSH-1 (population 60), as we could only obtain material fixed in 100\% ethanol, which is not well-suitable for histological studies.

Morphological analysis revealed that all individuals possessed most of the morphological and anatomical characteristics of $D$. subtentaculata s.l. as described by De Vries (1986a). The length of live animals ranged from 0.5 to $2 \mathrm{~cm}$. All individuals had a head of a low triangular shape, with two eyes of the dugesiid type in the middle of the head (supernumerary eyes occurred in some individuals of several populations). The dorsal body surface was provided with a granular and mottled pigmentation, extending from anterior to the eyes to the posterior region, excepting the auricular grooves, which were free of pigment. Despite this broad agreement with the known external morphology of the species, we also recorded variable morphological characteristics not previously reported by De Vries (1986a). First, all analyzed individuals of PSH-2 and some populations of PSH-5 lacked dorsal pigmentation anterior to the eyes (Fig. 5). Second, two densely pigmented dorsal stripes were observed in all individuals of PSH-3 and PSH-4 (some individuals of PSH-2 also showed these stripes, although less evident). Further, all individuals of one population of PSH-5 (population 50) showed two weakly pigmented stripes on the ventral surface, only 


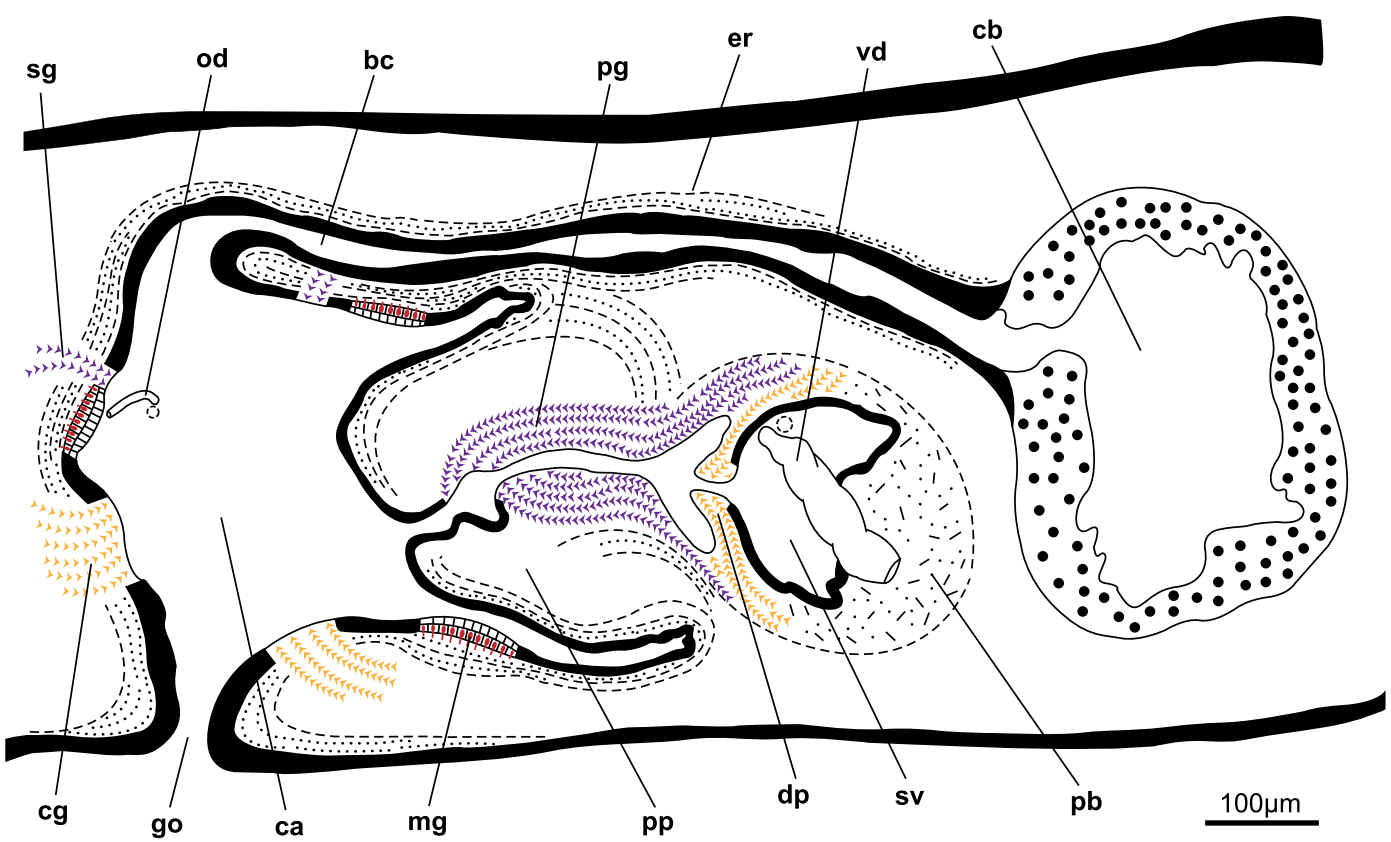

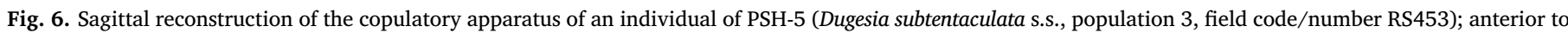
the right. Individuals of PSH-2 (D. aurea), PSH-3 (D. vilafarrei), and PSH-4 (D. corbata) basically show the same morphology.
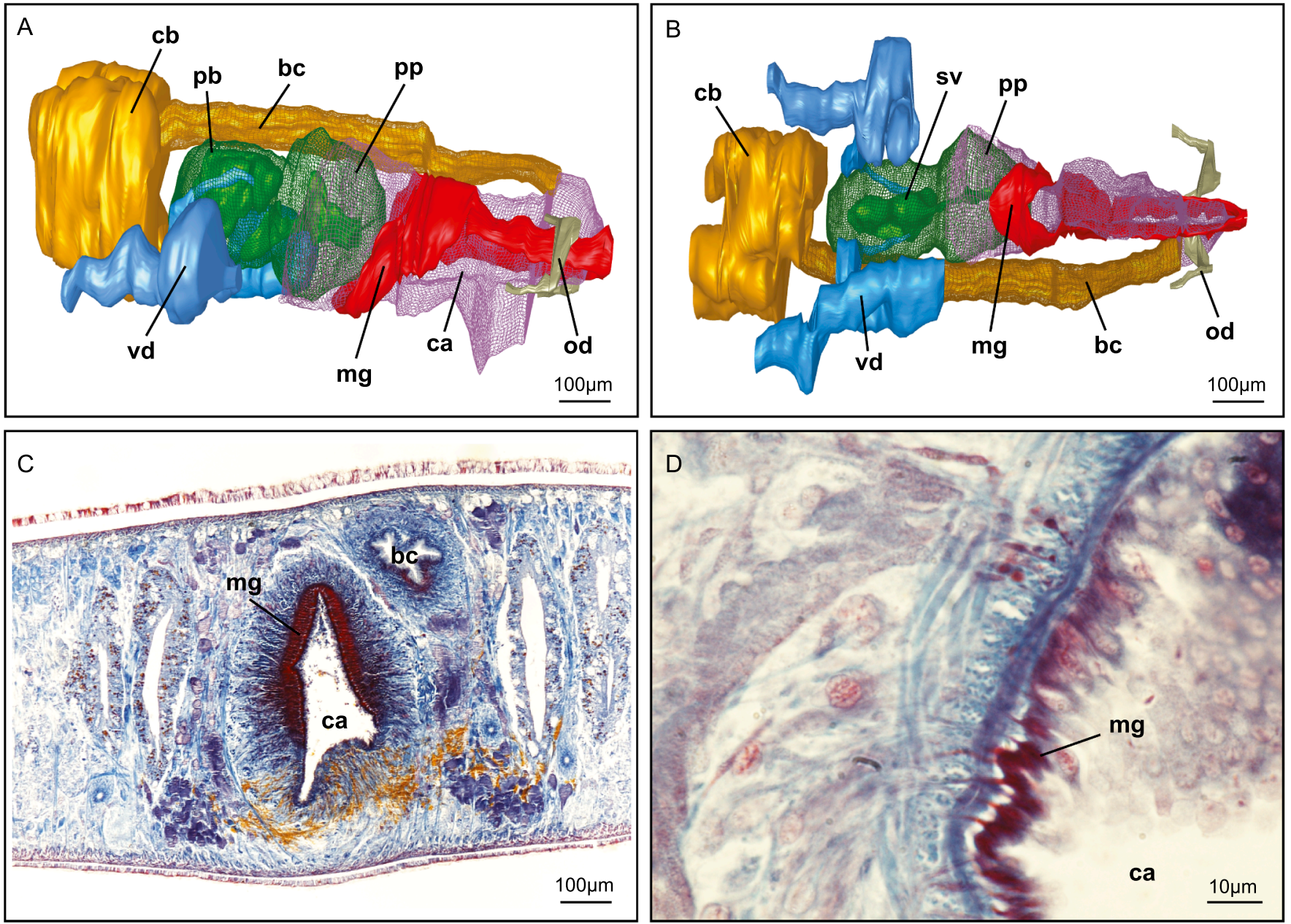

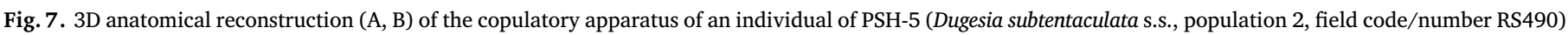

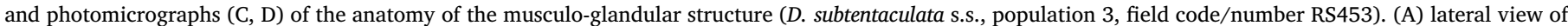

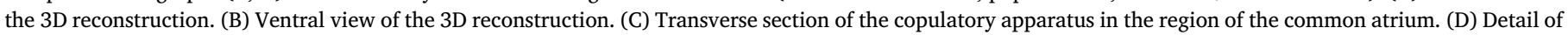
the pseudo-stratified epithelium of the ampulla-shaped cells that form the musculo-glandular structure. 
being visible when the individuals were observed in clove oil.

With respect to the anatomy of the copulatory apparatus, every individual from all PSHs showed the diagnostic combination of morphological characters of $D$. subtentaculata s.l. as described by De Vries (1986a) (Fig. 6): weakly muscular penis bulb, well delimited from a short and blunt penis papilla; central ejaculatory duct separated from a vesicle by a glandular valve-like diaphragm; abundant penial glands surrounding the seminal vesicle, the diaphragm and the ejaculatory duct. Besides the presence of abundant penial glands, bulb glands and shell glands, all individuals analyzed in this study possessed cement glands, discharging a yellowish secretion into the common atrium, surrounding the dorsal part of the gonoduct. Furthermore, in all specimens analyzed, a musculo-glandular structure in the atrium was present (mg in Fig. 6). Although this structure was mentioned by De Vries (1986a), she provided no detailed description of it. Examination of histological sections of the neotype of this species revealed that the glands were not well stained (all sections having an overall bluish colour), which possibly hindered the precise examination of this structure by De Vries (1986a). Our detailed examination of the histological sections of the new material, together with a 3D-reconstruction of the copulatory apparatus (Fig. 7), showed that this glandular structure extends on the major part of the atrial wall, from the ventral region under the penis papilla to the opening of the bursal canal into the atrium (Fig. 7A, B). This glandular region of the atrium showed a pseudostratified epithelium, strongly surrounded by muscles, through which an abundant erythrophilic secretion was discharged, thus making the cells look like red ampullae (Fig. 7C, D).

Despite all of these morphological resemblances, we found also some characters that differed between the various PSHs: (1) a parenchymatic ring at the base of the penis papilla was present in all individuals of PSH-4 and also in some specimens of PSH-5 (populations 17, 19, 30, and 40), (2) all individuals of PSH-2, PSH-3, PSH-4, and some individuals of PSH-5 (populations 30, 40, 41, 50, and 51) had a third layer of longitudinal muscles in the outer pharyngeal musculature, (3) all individuals of PSH-5 (excepting individuals of population 51) had elongated ovaries instead of rounded gonads, (4) all individuals of PSH-2 and population 51 of PSH-5 exhibited slightly asymmetrical openings of the vasa deferentia into the seminal vesicle; (5) in two populations of PSH-5 (populations 50 and 51) the ventral valve of the diaphragm was slightly smaller than the dorsal one. The first three of these morphological differences were already mentioned by De Vries (1986a) and were considered to be the result of intraspecific variation. The two last-mentioned differences are here reported for the first time. Finally, although PSH-5 showed some variable traits, each of these traits was shared with at least one of the other PSHs.

In conclusion, we did not find any stable diagnostic morphological feature for any of the various PSHs. Therefore, morphological data did not validate our Primary Species Hypotheses.

\subsubsection{Karyological validation}

Chromosomal measures of individuals from PSH-2, PSH-4, and population 46 of PSH-5 were compiled from Ribas (1990). We obtained new chromosomal measures of five individuals of PSH-3 and nine individuals of PSH-5, belonging to five different populations (Supplementary data S5). Additionally, we re-analyzed a metaphasic plate published by De Vries (1986b) concerning one individual from the type locality of D. subtentaculata s.l. (population 1 in the present study). As was the case also in the morphological analysis, we were not able to analyze any individual from PSH-1.

All individuals from PSH-2, PSH-3, and PSH-4 were diploid, with a chromosome complement of $2 \mathrm{n}=16$, while all populations analyzed of PSH-5 were triploid $(3 n=24)$, excepting individuals of population 7 , which were tetraploid $(4 \mathrm{n}=32)$, and population 51 that turned out to be diploid in a flux cytometry analysis performed in a parallel study (Leria et al., 2019). Relative length of the chromosomes was rather constant among the different PSHs (Supplementary data S12). On the other hand, our analysis revealed differences between the centromeric indices of the chromosomes among the various PSHs (Fig. 8; Supplementary data S12). All chromosomes of PSH-4 were metacentric, including four small supernumerary chromosomes. Individuals of PSH3 showed two sub-metacentric chromosomes (pairs 2 and 3), while specimens of PSH-2 had four sub-metacentric chromosomes (pairs 3, 4, 5 , and 6). In the case of PSH-5, several different aberrant chromosomes were present in each of the populations. Nevertheless, in all of them the first, sixth and eighth chromosome triplets turned out to be metacentric. The other chromosomes were either metacentric or sub-metacentric, depending on the population. Regarding the aberrant chromosomes, we identified a translocation already described by Ribas (1990), involving two chromosomes from the fourth and eighth triplet in all individuals analyzed of populations 4 and 22. Furthermore, a single large acrocentric chromosome was present in individuals from all populations, excepting population 46. This large acrocentric chromosome presumably belonged to the second triplet and its aberrant morphology possibly originated through translocation to a chromosome of the third triplet. Finally, we also found another putative translocation between one chromosome of the first triplet (donor) and one chromosome of the seventh triplet (receptor) (Fig. 8).

The karyological data described above reveal that the complements of PSH-2, PSH-3, and PSH-4 are clearly differentiated from each other and also from PSH-5. Moreover, despite the chromosomal variation detected among PSH-5 populations, all share several chromosomal characteristics that are different from PSH-2, PSH-3, and PSH-4. Therefore, karyological data validated our Primary Species Hypotheses.

\subsection{Secondary Species Hypotheses}

Four out of the five PSHs validated as independent lineages by BPP applied on multilocus data were also validated by karyological data, viz. PSH-2, PSH-3, PSH-4, and PSH-5. Unexpectedly, the status of those four taxa could not be corroborated on the basis of morphological and anatomical data. Therefore, we do here consider these four PSHs within D. subtentaculata s.l. to represent four different species that are cryptic at the morphological level (see Fig. 2). Three out of these four species are here described as new and thus receive a new specific epithet (see below). The name $D$. subtentaculata sensu stricto (further below referred to as D. subtentaculata s.s.) is retained for PSH-5, as it includes the population from the type locality of the species; the species is herein redescribed in order to account for the intraspecific variability as found in the present study. Although for PSH-1 we did not have information on either morphology or karyology, it was validated, nevertheless, by multilocus molecular data. Therefore, we considered PSH-1 to represent an unconfirmed candidate species of Dugesia, awaiting further morphological and karyological data that may test its taxonomic status.

\subsection{Taxonomic Section}

Order Tricladida Lang, 1884

Family Dugesiidae Ball, 1974

Genus Dugesia Girard, 1850

Dugesia subtentaculata s.s. (Draparnaud, 1801)

Material examined: Individuals from populations 1-46, 49-52, 54-59, and 61-63. GenBank accession numbers are detailed in Appendix A, codes (field numbers) of the individuals analyzed histologically are detailed in Supplementary data S4, while codes of the individuals analyzed karyologically are specified in Supplementary data S5. Morphological paratypes: individuals RS453 and RS474. 
PSH Pop. Karyotype

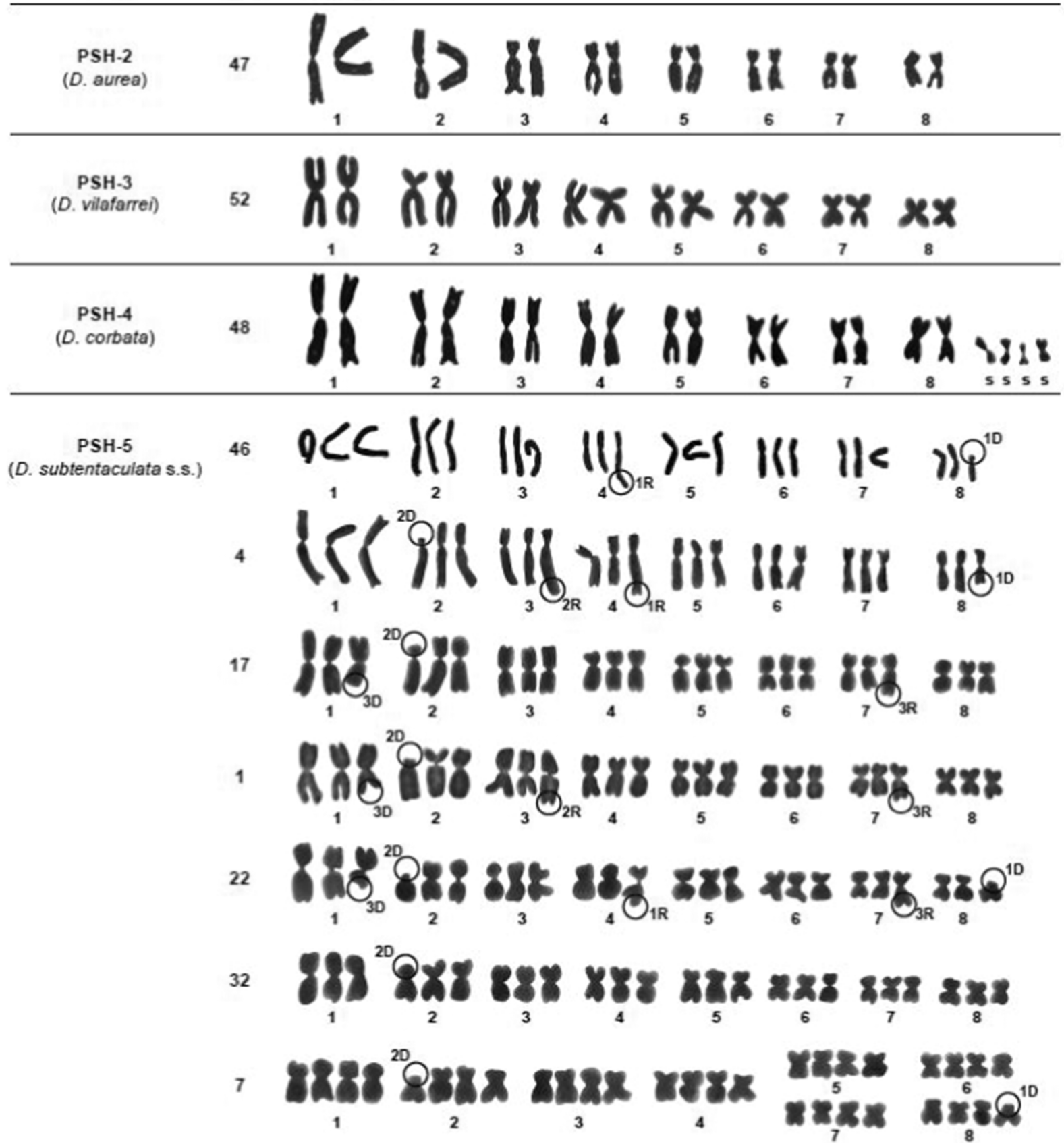

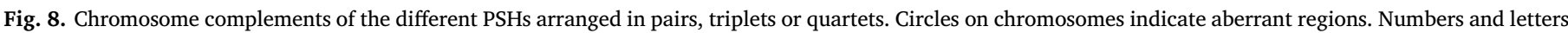

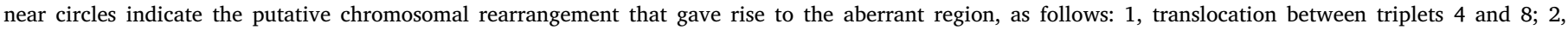

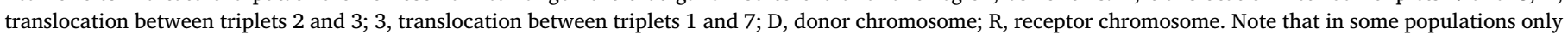
the donor or the receptor chromosomes of these putative translocations were detected. s: supernumerary chromosomes.

Karyological paratypes: individuals P6 and C12.3. DNA vouchers and chromosome slides were deposited in Dpt. de Genètica, Microbiologia i Estadística; Facultat de Biologia; Universitat de Barcelona, Barcelona, Spain. Histological sections were deposited in the collections of Naturalis Biodiversity Center, Leiden, the Netherlands.

Diagnosis: Molecularly, Dugesia subtentaculata s.s. comprises individuals that are identified as a single evolutionary unit together with individuals from populations $1-46,49-52,54-59$, and 61-63, when using the coalescence-based method BPP with the loci and settings detailed in the present study. Karyology: $2 \mathrm{n}=16$ (populations 50 and 51; Leria et al., 2019), $3 \mathrm{n}=24$, and $4 \mathrm{n}=32$ (population 7); chromosomes 1, 6, and 8 metacentric; chromosomes 2, 3, 4, 5, and 7 either metacentric or sub-metacentric, depending on the population; putative presence of aberrant chromosomes in all triplets, excepting the fifth and the sixth. In the present study corresponding to PSH-5.

Morphological re-description: Length of live animals ranges from 0.5 to $2 \mathrm{~cm}$. Head of a low triangular shape, with two eyes of the dugesiid type. Dorsal body surface mottled brownish or greyish, under 
natural conditions (Fig. 5); ventral surface pale or only lightly pigmented (population 50); auricular grooves devoid of pigmentation. Bilayered or three-layered (populations $30,40,41,50$, and 51) outer pharynx musculature.

Numerous dorsal testes, full of sperm, extending from the level of the ovaries to the posterior end of the body. A pair of rounded or slightly elongated ovaries situated approximately at one-third to onequarter of the distance between the brain and the root of the pharynx.

Anatomy of the copulatory apparatus characterized by: erythrophilic musculo-glandular structure covering most part of the wall of the common atrium; weakly muscular penis bulb, well-delimited from a short and blunt penis papilla; a parenchymatic ring at the base of the penis papilla present in some populations $(17,19,30$, and 40$)$; central ejaculatory duct (in population 49 slightly ventral), separated from a vesicle by a glandular valve-like diaphragm; abundant penial glands surrounding seminal vesicle, diaphragm and ejaculatory duct; abundant cement glands surrounding the dorsal region of the gonoduct; symmetrical or slightly asymmetrical (population 51) openings of the vasa deferentia into the posterior part of seminal vesicle; symmetrical openings of the oviducts into the posterior part of the atrium, just below the opening of the bursal canal into the atrium; bursal canal with ectal reinforcement all along the canal or at least extending from the atrium to the level of the penis bulb (Fig. 6).

Distribution: Southern France, Iberian Peninsula, and Northern Africa.

Reproduction: sexual, fissiparous, or alternation between reproductive strategies (i.e., facultative reproduction).

Dugesia aurea Leria, sp. nov.

Material examined: Individuals 47.1 to 47.5 preserved in ethanol $100 \%$ (GenBank accession numbers in Appendix A) and four samples fixed in Steinmann's fluid and, subsequently, preserved in $70 \%$ ethanol (codes/field numbers in Supplementary data S4). DNA holotype: individual 47.1, Soller, Mallorca, Balearic Islands, Spain, $39.75693 \mathrm{~N}$ 2.71193E. DNA paratypes: individuals 47.2 and 47.3. Morphological paratypes: individuals RS456 and RS458.1. DNA vouchers were deposited in Dpt. de Genètica, Microbiologia i Estadística; Facultat de Biologia; Universitat de Barcelona, Barcelona, Spain. Histological sections were deposited in the collections of Naturalis Biodiversity Center, Leiden, The Netherlands. Karyological information was extracted from Ribas (1990).

Etymology: The specific epithet refers to the etymology of the locality of Soller in Mallorca, which means "golden valley", supposedly derived from a term used by the Arabians when they arrived in this valley and saw the abundance of lemon trees. The name was also chosen to refer the gold-like coloration of the animals under natural conditions.

Diagnosis: Molecularly, Dugesia aurea comprises individuals that are identified as a single evolutionary unit together with individuals 47.1 to 47.5 , when using the coalescence-based method BPP with the loci and settings detailed in the present study. Karyology: $2 n=16$; chromosome pairs 1, 2, 7, and 8 metacentric; chromosome pairs 3, 4, 5, and 6 sub-metacentric. In the present study corresponding to PSH-2.

Morphological description: Length of live animals ranging from 0.5 to $1.5 \mathrm{~cm}$. Head of a low triangular shape, provided with two eyes of the dugesiid type in the middle of the head. Dorsal surface with goldlike coloration under natural conditions, with the pigmentation being granular and mottled, extending from the eyes to the posterior region (Fig. 5). In some individuals two dorsal pigmented stripes just being visible; auricular grooves devoid of pigmentation. Three-layered outer pharynx musculature. Numerous dorsal testes, full of sperm, extending from the level of the ovaries to the posterior end of the body. A pair of rounded ovaries situated approximately at one-third to one-quarter of the distance between the brain and the root of the pharynx. Reproductive apparatus as in Dugesia subtentaculata s.s. (see above) (Fig. 6).

Ecology and distribution: The species is known only from one site at Soller, Mallorca. Altitude: approx. $103 \mathrm{~m}$ a.s.l.

Reproduction: sexual.

Dugesia corbata Leria, sp. nov.

Material examined: Individuals 48.1 to 48.5 preserved in ethanol $100 \%$ (GenBank accession numbers in Appendix A) and three samples fixed in Steinmann's fluid and, subsequently, preserved in $70 \%$ ethanol (codes/field numbers in Supplementary data S4). DNA holotype: individual 48.1, Sa Calobra, Mallorca, Balearic Islands, Spain, $39.82932 \mathrm{~N}$ 2.81538E. DNA paratypes: individuals 48.2 and 48.3. Morphological paratypes: individuals RS461 and RS463. DNA vouchers were deposited in Dpt. de Genètica, Microbiologia i Estadística; Facultat de Biologia; Universitat de Barcelona, Barcelona, Spain. Histological sections were deposited in the collections of Naturalis Biodiversity Center, Leiden, The Netherlands. Karyological information extracted from Ribas (1990).

Etymology: The specific epithet refers to the locality where the species was found, which in Mallorca is known as "Nus de sa corbata" (in Catalan literally meaning necktie knot) because of the sharp bend in the road. The species name alludes also to the characteristic "necktieshape" of the Dugesia individuals.

Diagnosis: Molecularly, Dugesia corbata comprises individuals that are identified as a single evolutionary unit together with individuals 48.1 to 48.5 , when using the coalescence-based method BPP with the loci and settings detailed in the present study. Karyology: $2 n=16$ plus 4 supernumerary chromosomes; all chromosome pairs metacentric. In the present study corresponding to PSH-4.

Morphological description: Length of live animals ranging from 0.5 to $1.5 \mathrm{~cm}$. Head of a low triangular shape with two eyes of the dugesiid type in the middle. Dorsal body surface under natural conditions mottled dark reddish-brown, the granular pigmentation extending from anterior to the eyes to the posterior end of the body. With two densely pigmented broad stripes, running approximately from the region where the ovaries are located to the posterior region of the copulatory apparatus (Fig. 5). Auricular grooves devoid of pigmentation. Three-layered outer pharynx musculature. Numerous dorsal testes, full of sperm, extending from the level of the ovaries to the posterior end of the body. A pair of rounded ovaries situated approximately at one-third to one-quarter of the distance between the brain and the root of the pharynx. Reproductive apparatus as in Dugesia subtentaculata s.s. (see above) (Fig. 6).

Ecology and distribution: The species is known only from one site near Sa Calobra, in Mallorca. Altitude: $665 \mathrm{~m}$ a.s.l.

Reproduction: sexual.

Dugesia vilafarrei Leria, sp. nov.

Material examined: Individuals 52.1, 52.4-52.6, 52.14, 53.1, 53.2, 54.1 , and 54.2 preserved in ethanol 100\% (GenBank accession numbers detailed in Appendix A) and two samples fixed in Steinmann's fluid and, subsequently, preserved in $70 \%$ ethanol (codes/field numbers in Supplementary data S4). DNA holotype: individual 52.14, El Bosque, Andalucía, Spain, 36.76123 N 5.50581 W. DNA paratypes: individuals 52.5 and 54.1. Morphological paratypes: individuals MV04-6 and MV08-1. Karyological paratypes: individuals Ind1 and Ind2. DNA 
vouchers and chromosome slides were deposited in Dpt. de Genètica, Microbiologia i Estadística; Facultat de Biologia; Universitat de Barcelona, Barcelona, Spain. Histological sections were deposited in the collections of Naturalis Biodiversity Center, Leiden, The Netherlands.

Etymology: The species epithet honours collaborator and co-author of the present study Dr. Miquel Vila-Farré, who discovered two out of the three populations of this species and has extensively contributed to our knowledge of planarians in the Iberian Peninsula.

Diagnosis: Molecularly, Dugesia vilafarrei is constituted by individuals that are identified as a single evolutionary unit together with individuals 52.1, 52.4-52.6, 52.14, 53.1, 53.2, 54.1, and 54.2, when using the coalescence-based method BPP with the loci and settings detailed in the present study. Karyology: $2 \mathrm{n}=16$; chromosome pairs 1 , 4, 5, 6, 7, and 8 metacentric; chromosome pairs 2 and 3 submetacentric. In the present study corresponding to PSH-3.

Morphological description: Length of live animals ranging from 0.5 to $1.5 \mathrm{~cm}$. Head of a low triangular shape, in the middle with two eyes of the dugesiid type. Dorsal body surface under natural conditions mottled brownish and provided with two broad and densely pigmented stripes, extending from the region where the ovaries are located to the posterior region of the copulatory apparatus (Fig. 5); auricular grooves devoid of pigmentation. Three-layered outer pharynx musculature. Numerous dorsal testes, full of sperm, extending from the level of the ovaries to the posterior end of the body. A pair of rounded ovaries situated approximately at one-third to one-quarter of the distance between the brain and the root of the pharynx. Reproductive apparatus as in Dugesia subtentaculata s.s. (see above) (Fig. 6).

Ecology and distribution: The species is only known from three localities in the Sierra de Grazalema (Andalusia, Spain), where it coexists with $D$. subtentaculata s.s. (in localities 52 and 54). Although these localities are geographically very close to each other, populations 52 and 53 belong to the fluvial basin of the river Guadalete (Atlantic watershed), whereas population 54 lives in the fluvial basin of the river Guadiaro (Mediterranean watershed). Altitude of the localities: $270 \mathrm{~m}$ a.s.l. (population 52); $427 \mathrm{~m}$ a.s.l. (population 53); $724 \mathrm{~m}$ a.s.l. (population 54).

\section{Reproduction: Sexual.}

\section{Morphological comparative discussion}

De Vries (1986a) did amply show and discuss that the reproductive anatomy of $D$. subtentaculata s.l., particularly its copulatory apparatus, stands apart from all other species of Dugesia and thus facilitates its discrimination. Evidently, the present study revealed that cryptic diversity is hidden underneath the similar morphology of the various $D$. subtentaculata s.l. populations, resulting in the recognition of three new species.

The musculo-glandular structure present in all analyzed individuals of the four species described in the present study is unique to these species. Although De Vries (1988a) described a musculo-glandular area also in the atrial wall of Dugesia debeauchampi De Vries, 1988, she mentioned that only in a restricted area some glands discharge into the atrium, which may imply that in this species extension of the musculoglandular zone is much more restricted than is the case in our four cryptic species. The parenchymatic ring of vacuolated tissue in the penis papilla of Dugesia corbata and some specimens of $D$. subtentaculata s.s. is present also in Dugesia leporii Pala, Stocchino, Corso \& Casu, 2000 and Dugesia liguriensis. In particular, the blunt penis papilla of $D$. leporii resembles the short penis of $D$. subtentaculata s.s., D. aurea, $D$. corbata, and $D$. vilafarrei. However, D. leporii differs from these last-mentioned four cryptic species in that its diaphragm is pointed, and that it has a dorsal penial valve (Pala et al., 2000; Stocchino et al., 2017). Dugesia liguriensis has a pointed diaphragm, in contrast to the valve-like diaphragm of $D$. subtentaculata s.s., D. aurea, D. corbata, and D. vilafarrei, while it also possesses a cone-shaped penis papilla, which differs from the barrel-shaped papilla present in the last-mentioned four cryptic species (De Vries, 1988b).

\section{Discussion}

4.1. Molecular species delimitation in organisms with high intraindividual genetic diversity

Our study is the first in which information on mosaic intraindividual genetic diversity is used to infer molecular species boundaries (see Leria et al., 2019 for more information regarding mosaicism), while being evaluated against results obtained with datasets without this information. It is important to note that the new, exclusively sexual species ( $D$. aurea, $D$. corbata and $D$. vilafarrei), have been delimited by all molecular discovery methods used, with only two exceptions (Fig. 3). Although the sample sizes for these species may seem low (at minimum 2-3 individuals per species for all methods and 5 individuals in some datasets), we consider the number of individuals analyzed sufficient for capturing the genetic variability of each species, since all of them are endemic to small geographic regions. In contrast, D. subtentaculata s.s., a species constituted by a large number of fissiparous and facultative populations (and only a few sexually reproducing ones), yielded different outcomes for candidate species, depending on the methods and datasets used.

From the three molecular delimitation methods applied for determining Primary Species Hypotheses, ABGD and GMYC were affected the most by inclusion or exclusion of intraindividual genetic data. For both molecular markers, when ABGD was applied to the datasets with intraindividual information, it delimited as the same candidate species those haplotypes that had been delimited as different candidate species in the non-cloned datasets. A putative explanation for this different behaviour of ABGD may be that the non-cloned datasets contained ambiguous sites, whereas in the cloned datasets all haplotypes were resolved. It has been suggested that ambiguous sites may directly influence calculation of the genetic distances among sequences, while they may bias also the topology and branch lengths of Maximum Likelihood and Bayesian phylogenetic reconstructions (Lemmon et al., 2009). Therefore, a high number of ambiguous sites in the non-cloned datasets may have resulted in an increase of genetic distances and, thus, in a consequent over-splitting of candidate species when ABGD was applied.

In two cases, GMYC failed to return significant results. For Cox1, the method failed to delimit species in the dataset with intraindividual information, while in the case of Dunuc12 it was the other way around. This lack of significant results in GMYC could be due to the fact that the trees based on these datasets showed a more regular branching pattern, which possibly hindered GMYC to detect a clear transition from speciation to coalescence. Nonetheless, in the two other cases a delimitation scheme was obtained, but then GMYC resulted in an over-splitting of D. subtentaculata s.s. and even of one of the sexual species (D. aurea). In both cases, GMYC delimited as different putative species haplotypes of the same individual. This tendency of GMYC in over-splitting candidate species has been documented for both empirical and simulated data and has been principally attributed to gene flow or incomplete lineage sorting (Luo et al., 2018; Talavera et al., 2013). Our present study shows that high intraindividual diversity due to fissiparous reproduction may cause similar over-splitting effects in the performance of GMYC.

In contrast to ABGD and GMYC, the mPTP method delivered much more stable results across the four different datasets analyzed. Although mPTP uses substitution rates and tree topology to distinguish between intraspecies and interspecies processes (which may be influenced also by the number of ambiguous sites), the ability of this method to accommodate distinct rates of molecular evolution across linages may be the reason of its consistency.

All these findings indicate that for performing molecular species delimitation in Dugesia species (irrespective of their reproductive 
strategy, but particularly when they show fissiparous populations, such as D. subtentaculata s.s.), it is very important to include intraindividual genetic diversity, as the haplotypes of some individuals may be so different that some methods may identify these as belonging to separate putative species. However, when intraindividual data is not available, we recommend mPTP as the preferred method and to avoid ABGD or GMYC, as mPTP delivers more conservative results and thus minimizes over-splitting of putative candidate species. This may apply not only to other planarian genera but also to other asexual organisms with presumably high intraindividual genetic diversity due to asexual reproduction, such as starfishes, ribbon worms, or stick insects, among many others (Ament-Velásquez et al., 2016; Bast et al., 2018; GarciaCisneros et al., 2015).

\subsection{Multilocus data in Dugesia systematics: A useful addition}

Incorporation of the six new nuclear markers (Dunucs), the ribosomal gene $28 \mathrm{~S}$, and three small mitochondrial fragments, has greatly improved the resolution of the phylogenetic relationships among the different cryptic species previously subsumed under $D$. subtentaculata s.l., when compared to results obtained previously with single locus phylogenies (Lázaro et al. 2009). The species tree generated in the present study (Supplementary data S11) corroborated that individuals of population 60 (PSH-1) constitute the sister-group of the rest of the species included in this analysis, as already pointed out by Solà (2014). Moreover, this species tree revealed that the two new species from Mallorca ( $D$. aurea and $D$. corbata) constitute a highly supported monophyletic group and that $D$. subtentaculata s.s. is the sister species of D. vilafarrei, albeit with a posterior probability value of only 0.82 .

Furthermore, the use of new nuclear loci allowed us to apply a multilocus method to species delimitation based on coalescence (BPP), which highly increased the confidence in our delimitation scheme. BPP has already proved to be very useful in delimiting species in various groups of land planarians (Carbayo et al., 2016; Mateos et al., 2017). Therefore, the addition of the new markers used in this study together with BPP, may form a very important and adequate tool to unravel the evolutionary history of some intricate groups within the genus Dugesia, such as species from the Aegean region (see below) (Sluys et al., 2013).

\subsection{Morphological crypsis in Dugesia}

The present study revealed the existence of four morphologically cryptic species within $D$. subtentaculata s.l. that are highly differentiated at both the molecular and karyological level. To the best of our knowledge, this represents the first case of morphologically cryptic species being described for the genus Dugesia. Nonetheless, possibly it may not form an isolated case. For example, in a taxonomic study of Dugesia in the Aegean region, several lineages were molecularly highly differentiated from each other (identified as independent linages by GMYC) but did not show any morphological differentiation and, therefore, they were considered as "Deep Conspecific Lineages" (DCL) (Sluys et al., 2013). However, it may well be that inclusion of additional sources of information besides DNA and morphology, such as, for example, chromosomal characteristics, will reveal cryptic Dugesia species being present also in that region.

Examples of morphological crypsis in planarians are already available, such as in the land planarians of the genus Obama Carbayo et al., 2013 (Álvarez-Presas et al., 2015), in which two pseudo-cryptic species were highly differentiated at molecular level but were indistinguishable by the commonly used morphological features. Further, putative cryptic diversity recently has been proposed also for the freshwater planarian species Polycelis coronata (Girard, 1891) (Rader et al., 2017) and Crenobia alpina (Dana, 1766) (Brändle et al., 2017) on the basis of the high genetic differentiation detected within these two species. Given the fact that planarians are frequently difficult to diagnose solely on the basis of morphological characters and that cryptic species are probably common in this group, the integrative taxonomic procedure applied in the present study (Fig. 2) could be highly useful for future taxonomic studies on planarians.

Cryptic diversity generally may be explained by three different mechanisms: (1) recent diversification, (2) morphological convergence or parallelism, or (3) morphological stasis (Fišer et al., 2018; Struck et al., 2018). In the first case, the differentiated lineages are at an early stage in the speciation continuum during which some differences in variable loci have accumulated but insufficient time has passed for the evolution of any morphological differences, as morphological characters generally are under the influence of multiple genes (Fisher, 1999). In order to test the hypothesis of recent diversification, it is necessary to put the group of interest in a temporal framework. A recent study on the historical biogeography of the genus Dugesia estimated the divergence between the unconfirmed candidate species PSH-1 and two populations of $D$. subtentaculata s.l. to date back to approximately 20-10 million years ago (Mya) (Solà, 2014). Although additional calibration studies would be needed, including individuals of the three new cryptic species herein described, the previously mentioned calibration analysis together with the high genetic and karyological differentiation among these cryptic species, strongly suggests that the lack of morphological differentiation among them may not be due to recent diversification.

On the other hand, the molecular monophyly of the four species suggests that morphological crypsis may not result from morphological convergence either, but it does not rule out parallelism. If parallelism would have been the underlying process, it would mean that the detailed characteristics of the copulatory apparatus in these species had evolved independently in each lineage. However, among the different features that characterize these species, there is one character that may be unique in Dugesia, viz. the musculo-glandular structure (mg in Fig. 7). The glandular component of this structure may be formed by shell glands. In most Dugesia species, shell glands discharge their secretion into the bursal canal around the oviducal openings or, less common, into another restricted region of the atrium, such as in Dugesia debeauchampi (De Vries, 1988a). But in all specimens of the four cryptic species analysed in the present study these massive, putative shell glands apparently have shifted, so that they discharge through the major part of the atrial wall, extending from dorsal to ventral surface. Therefore, the most parsimonious interpretation for the occurrence of this character state in these four cryptic species is that it evolved in their most recent common ancestor. Under this hypothesis, morphological parallelism would also be discarded as an explanation for the occurrence of morphological crypsis between these species.

Hence, only stasis remains as a plausible, alternative explanation for the phenomenon of morphological crypsis in these species. Morphological stasis in anatomical features of planarians has been proposed for South American species of the genus Girardia (Sluys et al., 2005). The genus Dugesia also exhibits a high degree of stasis in both external and anatomical features, notably the copulatory apparatus, despite the fact that the genus presumably is very old (dating back to about 240 Mya; Solà, 2014). An explanation for such a high degree of stasis might be that deviations from these morphological characteristics are under strong selective pressure (i.e., that these characters are subject to stabilizing selection), as has been proposed for other character complexes in cryptic or pseudo-cryptic species of sea urchins, unicellular algae, and lizards (Egea et al., 2016; Sáez et al., 2003; Smith et al., 2011). Importantly, the existence of morphological stasis in $\mathrm{Du}$ gesia, and very likely also in other planarian genera, suggests that the actual planarian species richness might be highly underestimated, since the anatomy of the copulatory apparatus has been and still is the principal criterion for delimiting species boundaries in these organisms.

Dugesia subtentaculata s.s. is the only species of this species complex that shows some noticeable intraspecific morphological variation. Although we cannot completely rule out that future analysis of additional material will also reveal some variability in $D$. aurea, D. corbata, 
and D. vilafarrei, current absence of morphological variation within these three species might be related to their very restricted distributions. As in the case of the molecular data, such restricted geographic ranges may signal population bottlenecks that resulted in a reduction of the morphological variability in each of these three species. However, it may be the case that the intraspecific variability in $D$. subtentaculata s.s. results from various factors that are mainly linked to differences in developmental stages or processes. For example, presence or absence of a parenchymal ring in the penis papilla, or minor changes in the shape of the ovaries, might be due to different stages in the reproductive cycle of the animal. Moreover, the development of the reproductive system might be different in specimens that build it once and retain it for long periods of time (sexual populations) versus those that alternate fission and sexual reproduction. At the present moment we can only speculate on the underlying mechanisms of this morphological variability in $D$. subtentaculata s.s. and its absence in $D$. aurea, D. corbata, and $D$. vilafarrei. However, this variability is restricted to minor variations in some morphological characteristics (e.g., slightly asymmetrical openings of the vasa deferentia into the seminal vesicle, or small differences in the extension of the ectal reinforcement) that presumably have little impact on the functionality of the copulatory apparatus, the structure of which is highly similar among the different populations of $D$. subtentaculata s.s. and between the different species of this species complex.

\subsection{Karyological variability in planarians}

Our karyological analysis revealed that the four cryptic species, namely $D$. aurea, $D$. corbata, $D$. vilafarrei and $D$. subtentaculata s.s., show different chromosomal features. The species $D$. aurea, $D$. corbata, and $D$. vilafarrei are all diploid $(2 \mathrm{n}=16)$ but can be distinguished from each other and from $D$. subtentaculata s.s. by the centromeric indices of several chromosome pairs; the last-mentioned species can be distinguished also from the others by the ploidy level (most populations being polyploid) and by the presence of aberrant chromosomes. Similar complex karyological situations as present in D. subtentaculata s.s., have been observed also in many other Dugesia species with both sexual and fissiparous populations, such as D. japonica, D. ryukyuensis, D. benazzii, and D. maghrebiana (Benazzi-Lentati and Benazzi, 1985; Stocchino et al., 2009; Tamura et al., 1998, 1991; Vacca et al., 1993). Moreover, high levels of karyological variability among closely related species have been reported for several planarian genera of the family Dugesiidae, such as Schmidtea Ball, 1974, Cura Strand, 1942, and Girardia Ball, 1974 (Benazzi and Puccinelli, 1973; Benya et al., 2007; Gourbault and Benazzi, 1975).

Girardia tigrina (Girard, 1850) presents a high copy number of mariner-like transposons (Garcia-Fernàndez et al., 1995), and the genome of Schmidtea mediterranea harbours a novel type of giant retroelements (Grohme et al., 2018). The presence in high amounts of such elements may be related to the high incidence of chromosome rearrangements observed in this group of animals, as has been shown also for other organisms (Feschotte and Pritham, 2007). Moreover, it has been suggested that fissiparous reproduction may allow planarians to endure situations of unbalanced karyotypes, as during periods of fissiparous reproduction individuals do not undergo meiosis and, thus, would not suffer from selection against chromosomal rearrangements (Leria et al., 2018). Thus, the high motility of genome elements of planarians may explain the karyological variability detected between the four cryptic Dugesia species described in the present study. Moreover, in the case of $D$. subtentaculata s.s. its mainly fissiparous type of reproduction may explain the high incidence of chromosome aberrations.

The different chromosome portraits in each of these four cryptic species possibly prevent them to successfully crossbreed, as in the offspring the different centromeric positions of several homologous chromosomes would generate anomalous chromosome pairings during meiosis, thus resulting in incorrect segregation or unbalanced gametes after recombination (Faria and Navarro, 2010). It may even be the case that these chromosomal differences actually were the drivers of the speciation process, in the same way as recently proposed for the genus Schmidtea (Leria et al., 2018). On the other hand, the chromosomal differences simply may be due to karyotype changes that have accumulated after the speciation process. For example, speciation of the Mallorca populations, resulting in $D$. aurea and $D$. corbata, may have resulted from geographic isolation from peninsular populations. In a similar way, the two species in Mallorca may have diverged because of geographic isolation, since $D$. aurea and $D$. corbata occur in the Torrent de Soller and Torrent de Sa Calobra-Pareis, respectively, which are two separate ancient fluvial basins of the Tramuntana Range (Silva et al., 2005). Thus, geographic isolation may have been an alternative driver of speciation, instead of karyological differences. In contrast, the speciation event that gave rise to D. vilafarrei and D. subtentaculata s.s., both species co-occurring at two localities in the Iberian Peninsula, possibly was due to a triploidization event in the ancestor of $D$. subtentaculata s.s. (Leria et al., 2019). This change from diploidy to triploidy possibly triggered the shift to fissiparous reproduction, thus immediately preventing the outcrossing of the original diploids with the newly formed fissiparous triploids. Therefore, it is possible that karyological plasticity did drive indeed speciation between $D$. vilafarrei and D. subtentaculata s.s.

\subsection{Conservation status of the species}

Three out of the four cryptic species described in the present study are endemic at very restricted geographic areas. Dugesia aurea and D. corbata meet the IUCN criterion of Critically Endangered (CR), since only a single locality of each is presently known (Section $\mathrm{V}$, point B.2.a), while D. vilafarrei meets the IUCN criterion of Endangered (EN) as it is presently known from less than five localities (Section V, point B.1.a) (IUCN, 2012). We consider that this assessment of the conservation status of these species represents their actual situation, since we performed many extensive samplings in the Balearic Islands and in the Iberian Peninsula over the past few years. As is the case with these species, many other species of the genus Dugesia, and also of other genera of freshwater planarians, are known only from a few localities and/or are subjected to habitat loss (see Lázaro et al., 2011; Sluys et al., 2013). Although freshwater planarians play an important role in trophic networks (they can act as top predators) (Teal, 1957; Tilly, 1968) and may be used as bioindicators of the quality of the water due to their sensitivity to pollutants (Knakievicz, 2014), no planarian species is currently included in any conservation policy, albeit some proposals have been made (e.g., Souza et al., 2016), which, unfortunately, holds true for most invertebrate species (Cardoso et al., 2011). We do here propose to include in the IUCN Red List the new species $D$. aurea and $D$. corbata under the conservation category of Critically Endangered (CR), and D. vilafarrei as Endangered (EN). It is our hope that then conservation policies will be formulated to protect these species and that this will form a precedent for many other conservation actions in planarians and invertebrate species in general.

\section{Acknowledgments}

We are grateful to Eduard Solà, Arnau Poch, Aleix Valls, Mette Handberg-Thorsager, José María Martín Durán, Albert Cardona and Francisco Monjo for their assistance during the fieldwork.

\section{Funding}

This research was supported by DGI-MEC projects CGL2011-23466 and CGL2015-63527 to MR. 


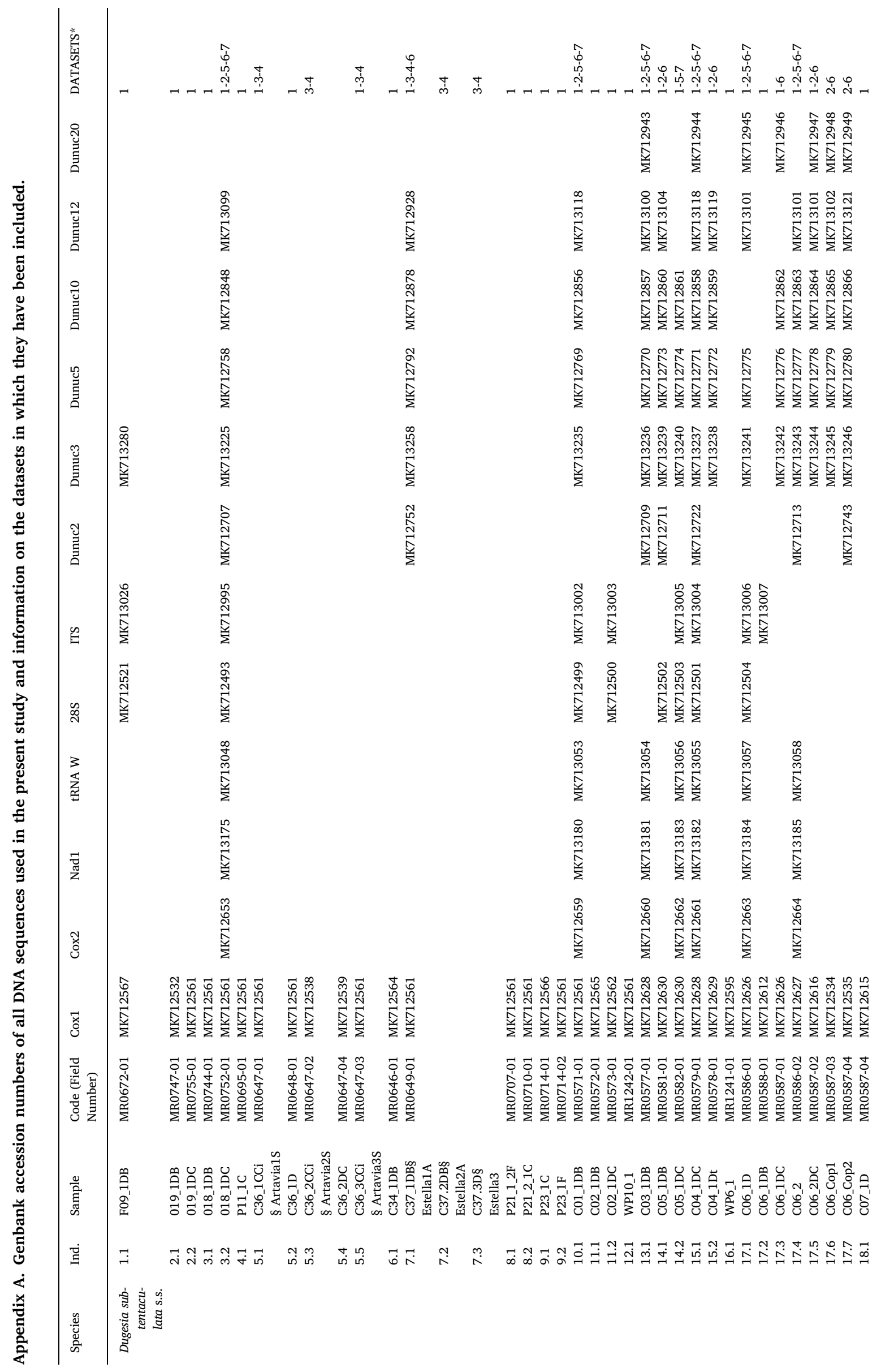




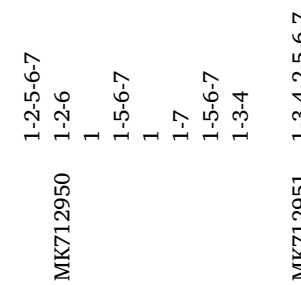

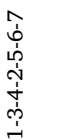

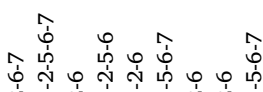

ஸ่

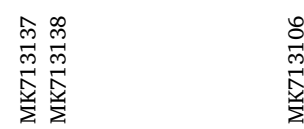

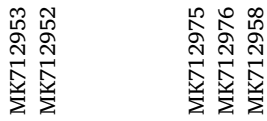

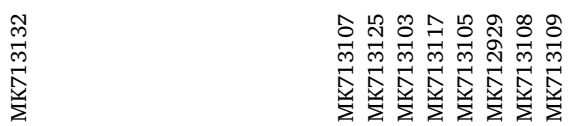

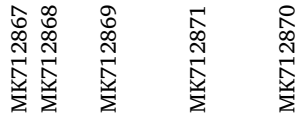

商

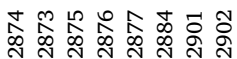

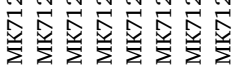

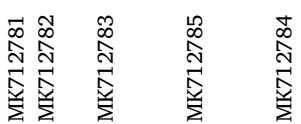

离

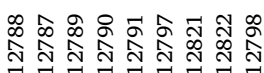

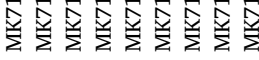

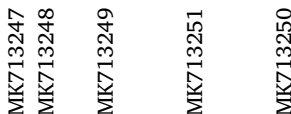

设

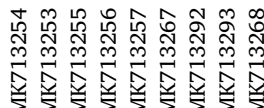

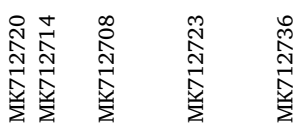

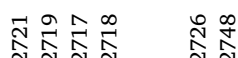

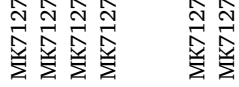

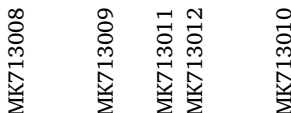

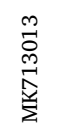

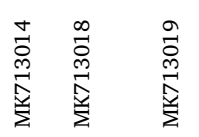

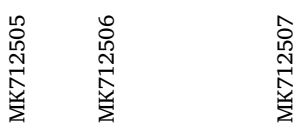

足

离

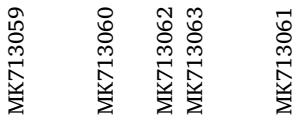

苍

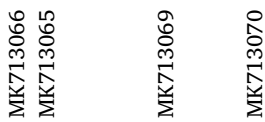

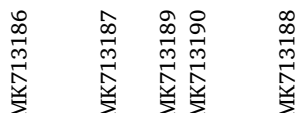

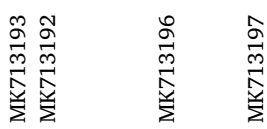

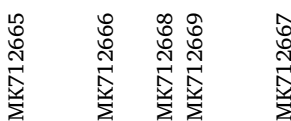

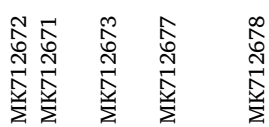

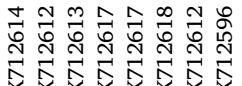

总

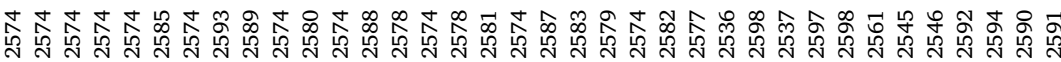

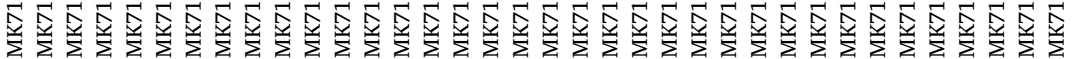

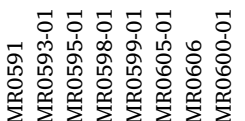

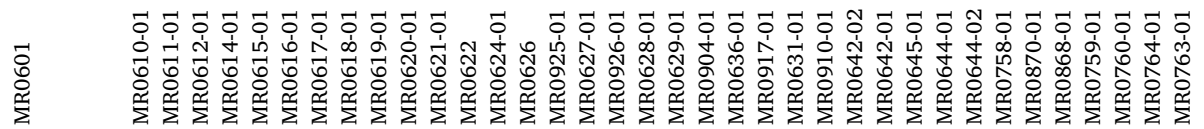

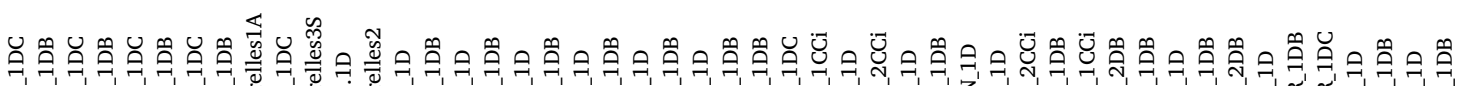

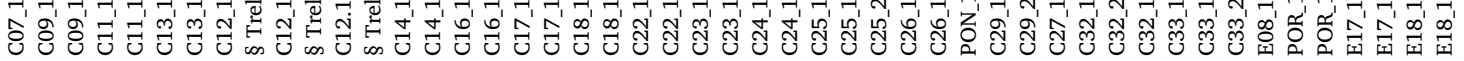

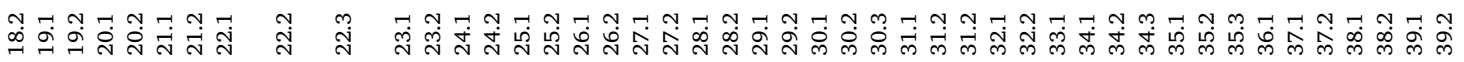




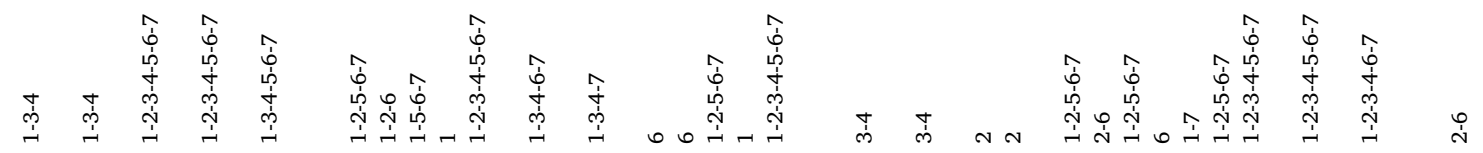

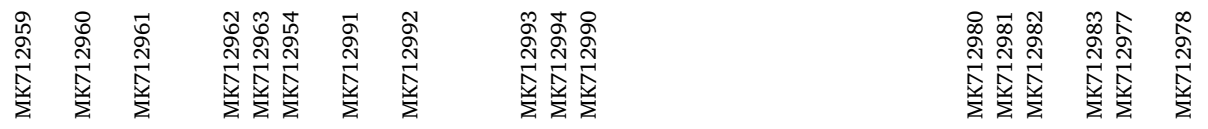

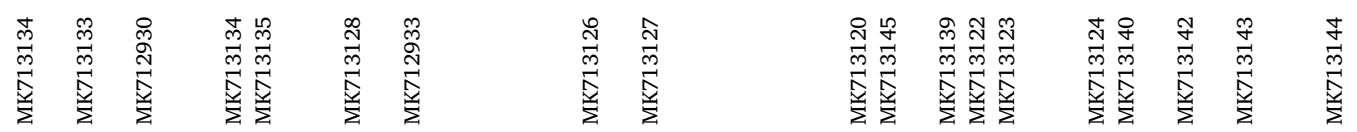

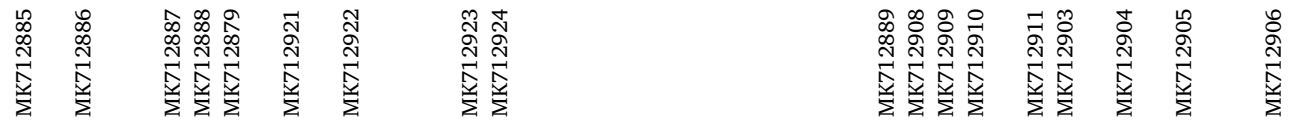

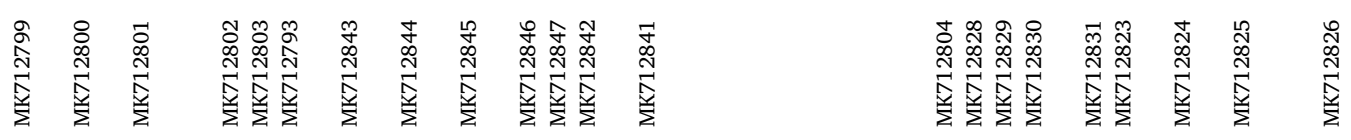

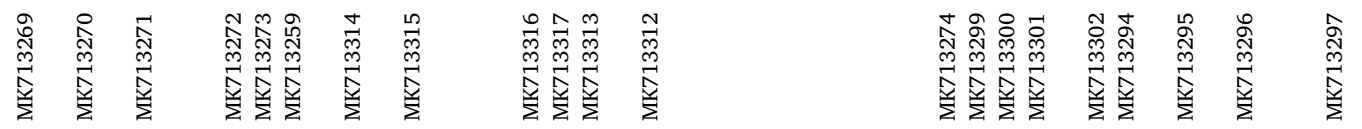

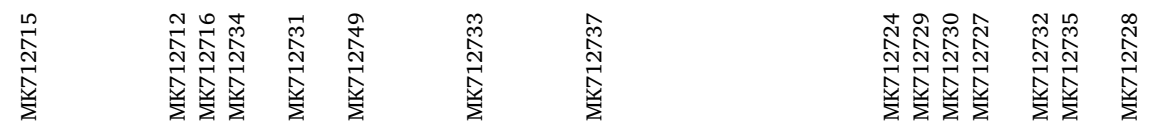

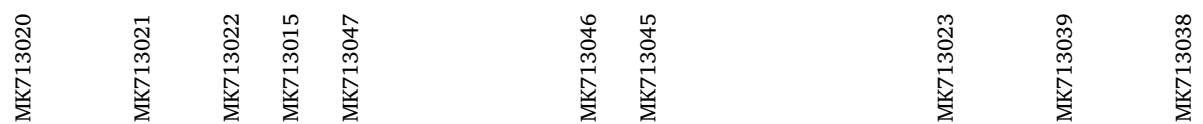

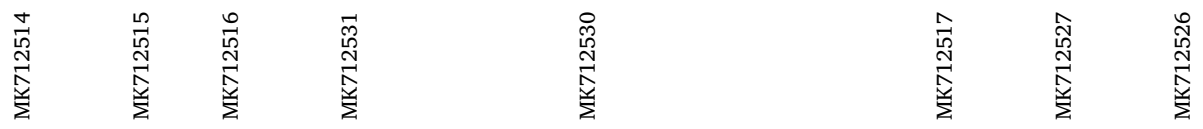

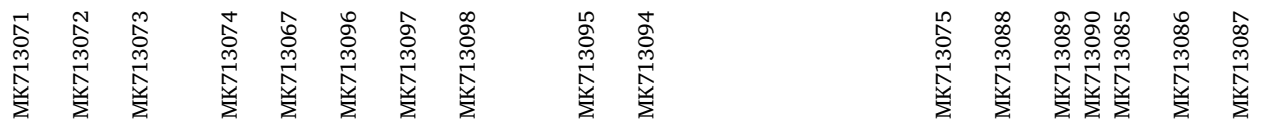

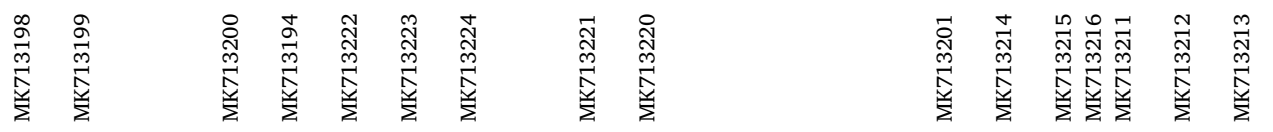

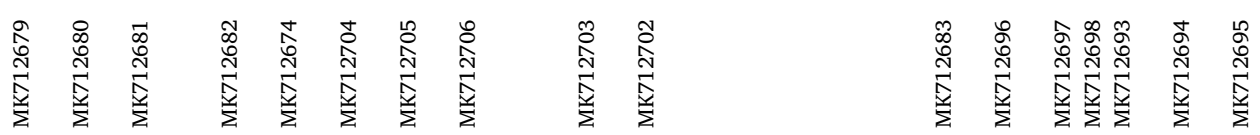

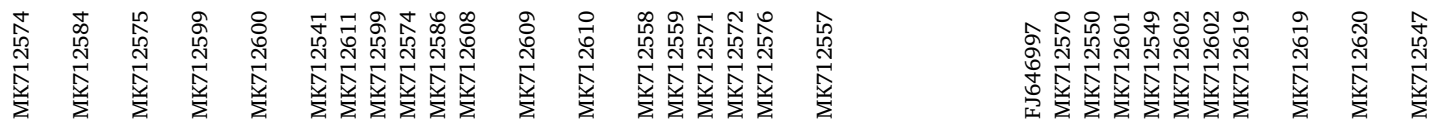

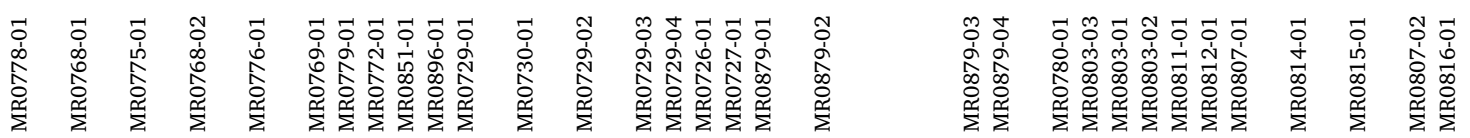

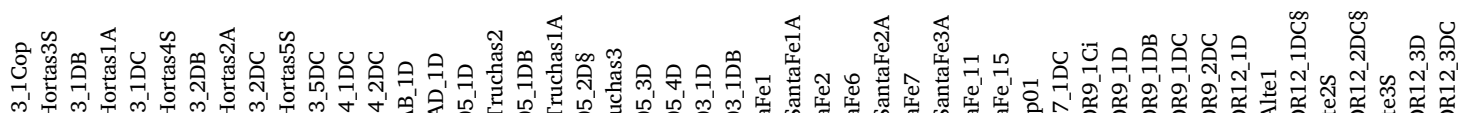

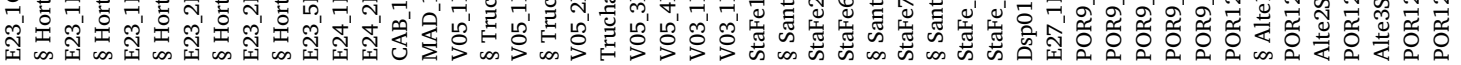

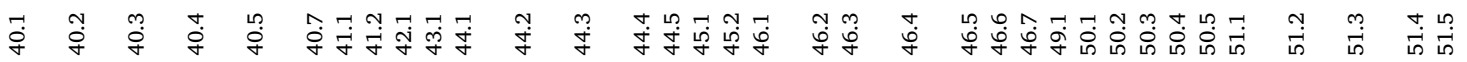




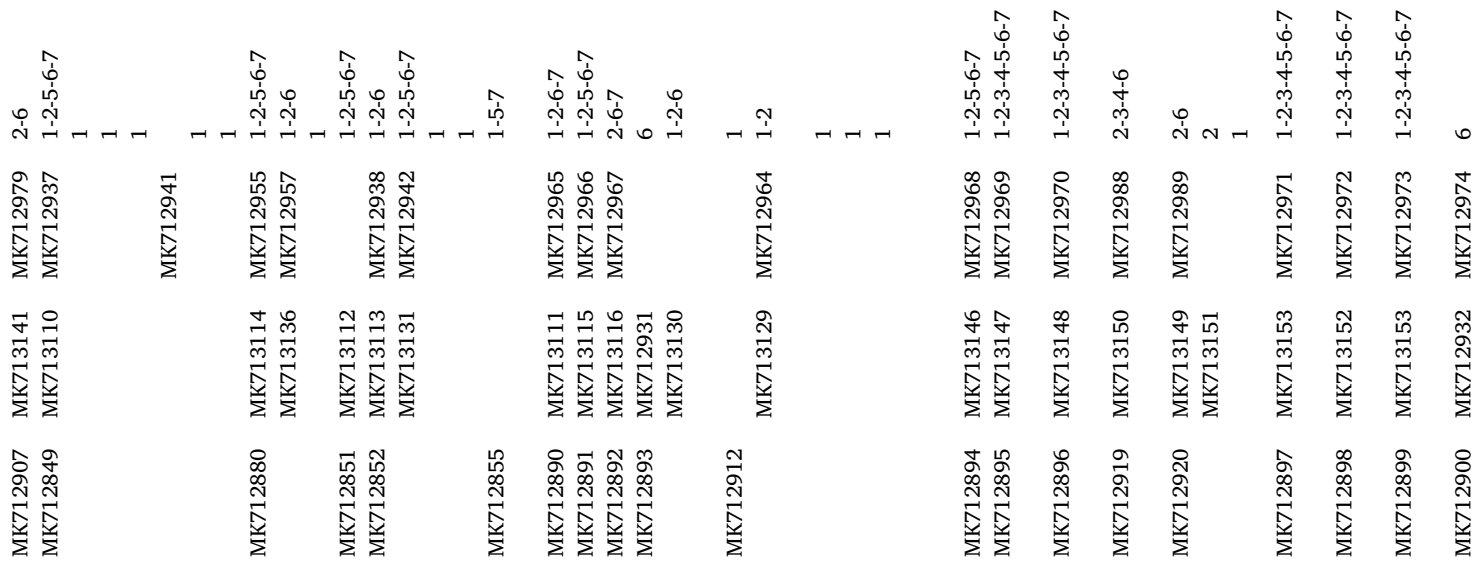

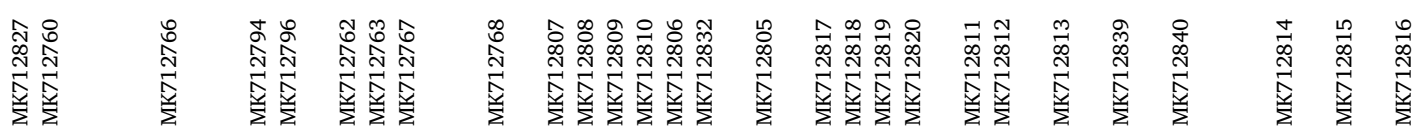

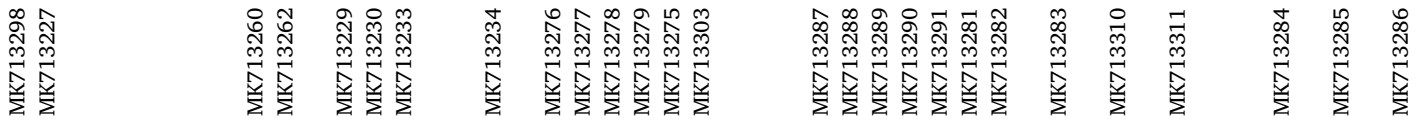

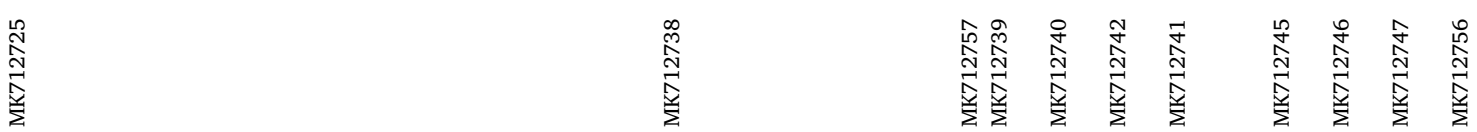

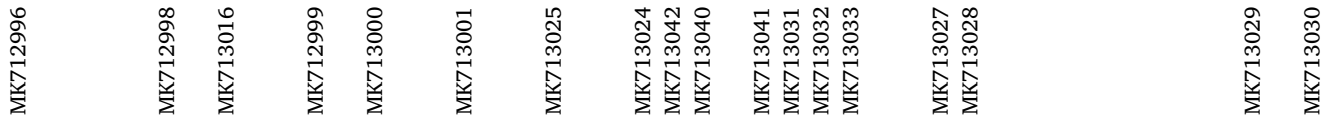

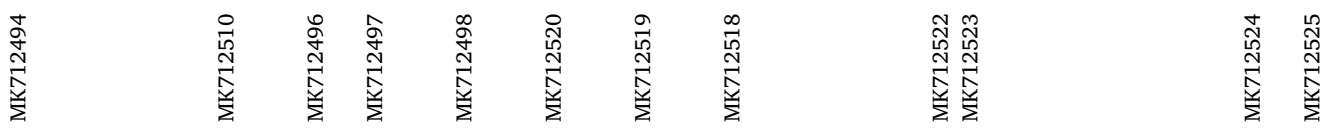

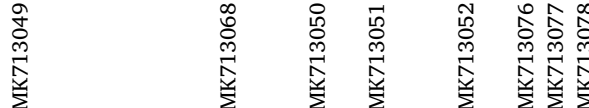

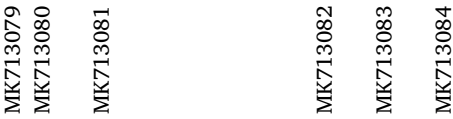

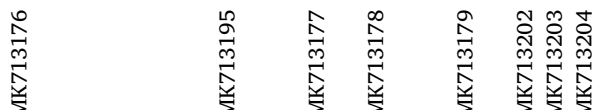

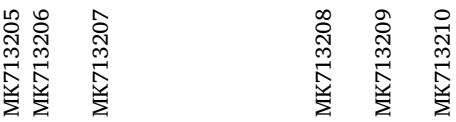

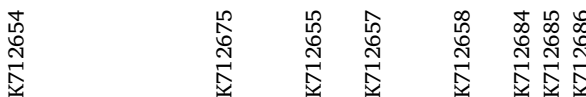

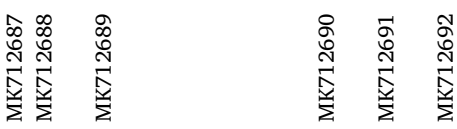

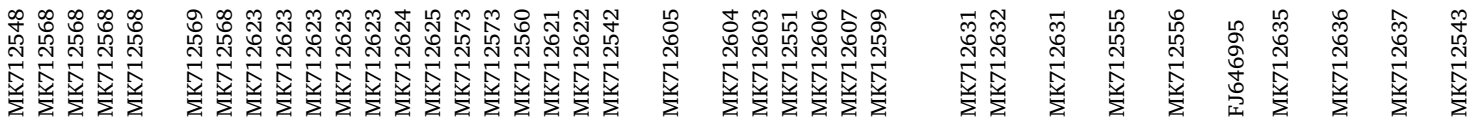

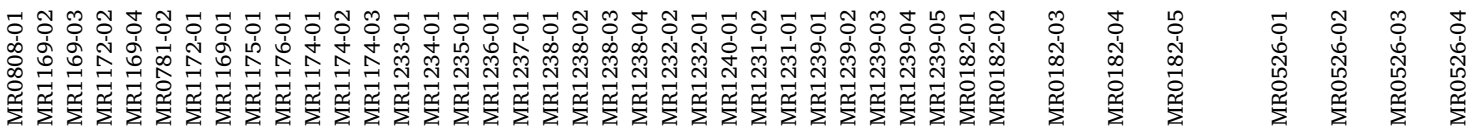

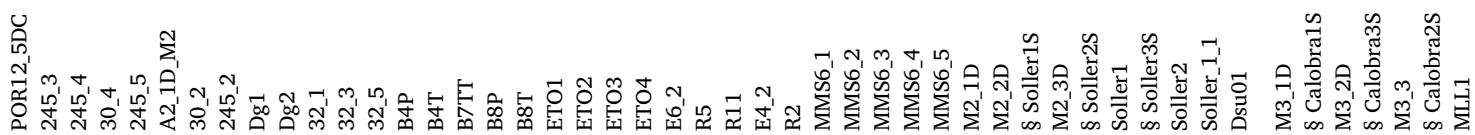

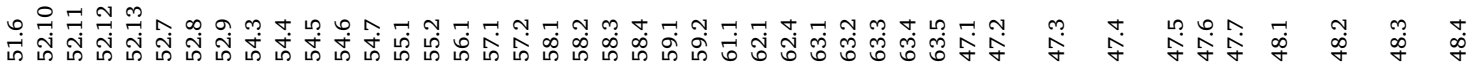




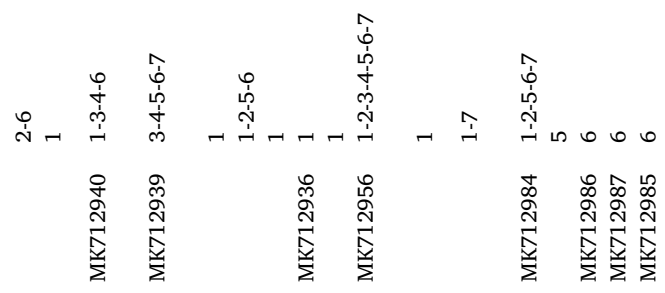

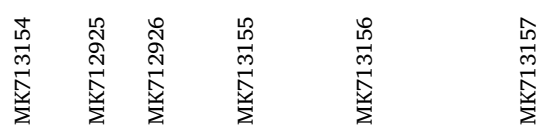

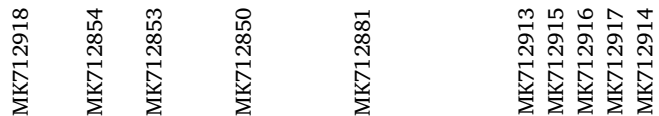

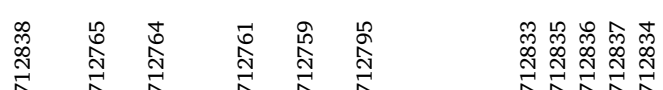

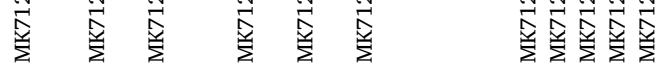

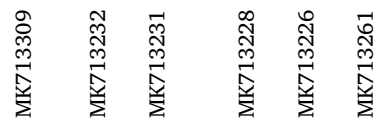

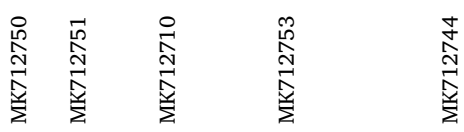

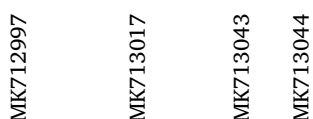

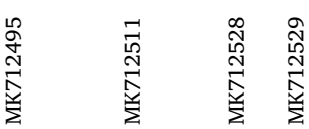

喜曼

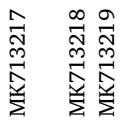

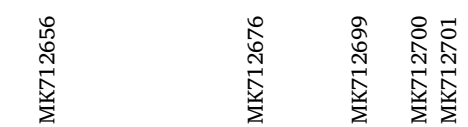

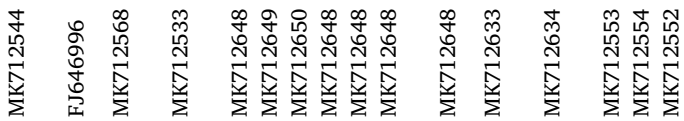

낭

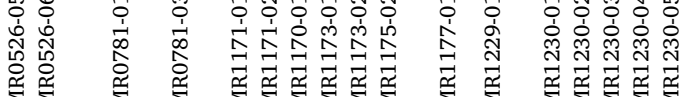

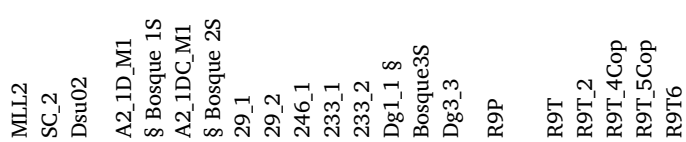

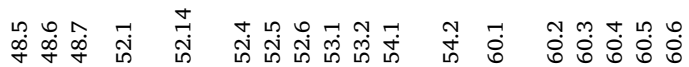

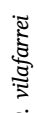

ji

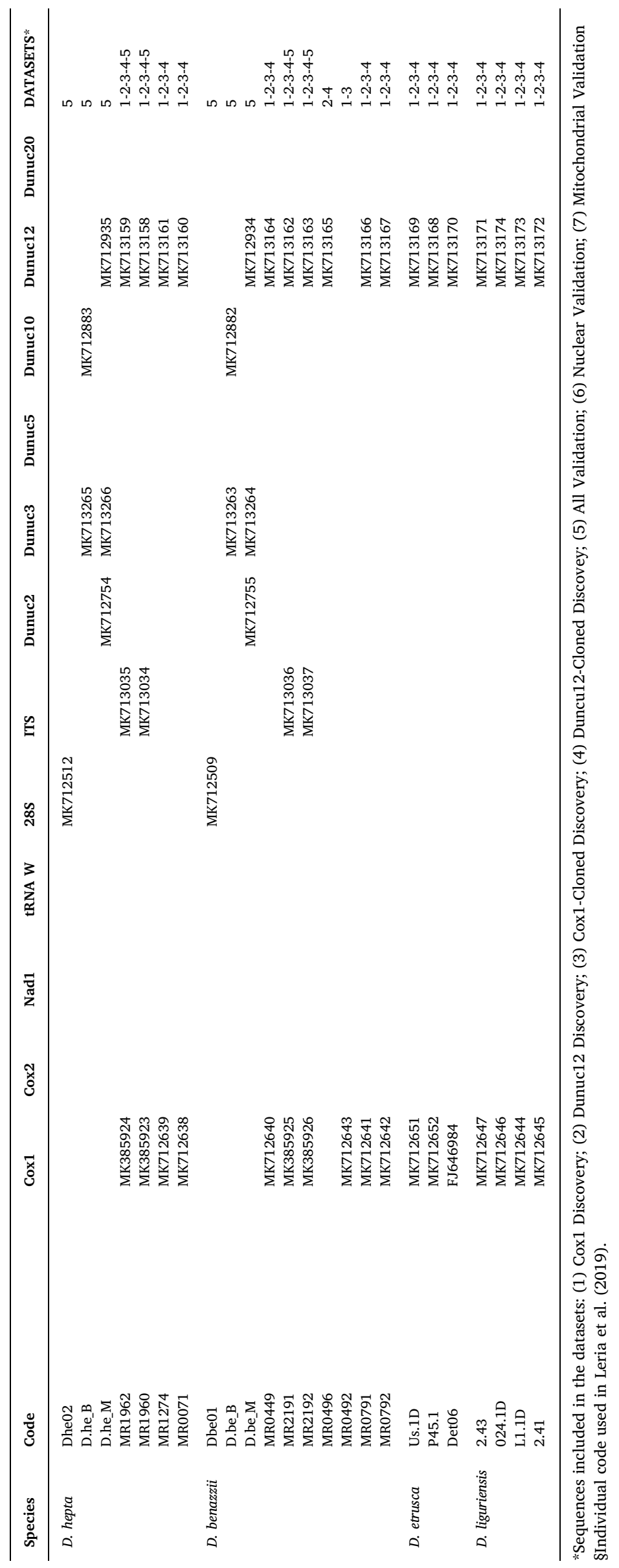




\section{Appendix B. Supplementary material}

Supplementary data to this article can be found online at https://doi.org/10.1016/j.ympev.2019.05.010.

\section{References}

Altınordu, F., Peruzzi, L., Yu, Y., He, X., 2016. A tool for the analysis of chromosomes: KaryoType. Taxon 65, 586-592. https://doi.org/10.12705/653.9.

Álvarez-Presas, M., Amaral, S.V., Carbayo, F., Leal-Zanchet, A.M., Riutort, M., 2015. Focus on the details: morphological evidence supports new cryptic land flatworm (Platyhelminthes) species revealed with molecules. Org. Divers. Evol. 15, 379-403. https://doi.org/10.1007/s13127-014-0197-z.

Álvarez-Presas, M., Baguñà, J., Riutort, M., 2008. Molecular phylogeny of land and freshwater planarians (Tricladida, Platyhelminthes): From freshwater to land and back. Mol. Phylogenet. Evol. 47, 555-568. https://doi.org/10.1016/j.ympev.2008. 01.032 .

Ament-Velásquez, S.L., Figuet, E., Ballenghien, M., Zattara, E.E., Norenburg, J.L., Fernández-Álvarez, F.A., Bierne, J., Bierne, N., Galtier, N., 2016. Population genomics of sexual and asexual lineages in fissiparous ribbon worms (Lineus, Nemertea): hybridization, polyploidy and the Meselson effect. Mol. Ecol. 25, 3356-3369. https:// doi.org/10.1111/mec.13717.

Andrey, P., Maurin, Y., 2005. Free-D: An integrated environment for three-dimensional reconstruction from serial sections. J. Neurosci. Methods 145, 233-244. https://doi. org/10.1016/j.jneumeth.2005.01.006.

Baguñà, J., Carranza, S., Pala, M., Ribera, C., Giribet, G., Arnedo, M.A., Ribas, M., Riutort, M., 1999. From morphology and karyology to molecules. New methods for taxonomical identification of asexual populations of freshwater planarians. A tribute to Professor Mario Benazzi. Ital. J. Zool, 207-214.

Ball, I.R., 1970. Freshwater triclads (Turbellaria, Tricladida) from the Oriental region. Zool. J. Linn. Soc. 49, 271-294.

Bast, J., Parker, D.J., Dumas, Z., Jalvingh, K., Tran Van, P., Jaron, K., Figuet, E., Brandt, A., Galtier, N., Schwander, T., 2018. Consequences of asexuality in natural populations: Insights from Stick insects. Mol. Biol. Evol. 35 (7), 1668-1677. https://doi.org/ 10.1093/molbev/msy058.

Benazzi-Lentati, G., Benazzi, M., 1985. On the origin and the true nature of the tetraploid asynaptic planarian called Benazzi's G biotype. Caryologia 38, 269-279. https://doi. org/10.1080/00087114.1985.10797750.

Benazzi, M., Puccinelli, I., 1973. A Robertsonian translocation in the fresh-water triclad Dugesia lugubris: karyometric analysis and evolutionary inferences. Chromosoma 40, 193-198. https://doi.org/10.1007/BF00321464.

Benya, E., Leal-Zanchet, A., Santos, W., Hauser, J., Erdtmann, B., 2007. Chromosome polymorphism and complements in populations of Girardia species (Platyhelminthes, Tricladida, Paludicola) from Southern Brazil. Brazilian J. Biol. 67, 951-955.

Brändle, M., Sauer, J., Opgenoorth, L., Brandl, R., 2017. Genetic diversity in the Alpine flatworm Crenobia alpina. Web Ecol. 17, 29-35. https://doi.org/10.5194/we-17-292017.

Carbayo, F., Álvarez-Presas, M., Jones, H.D., Riutort, M., 2016. The true identity of Obama (Platyhelminthes: Geoplanidae) flatworm spreading across Europe. Zool. J. Linn. Soc. 5-28. https://doi.org/10.1111/zoj.12358.

Cardoso, P., Erwin, T.L., Borges, P.A.V., New, T.R., 2011. The seven impediments in invertebrate conservation and how to overcome them. Biol. Conserv. 144, 2647-2655. https://doi.org/10.1016/j.biocon.2011.07.024.

Carstens, B.C., Pelletier, T.A., Reid, N.M., Satler, J.D., 2013. How to fail at species delimitation. Mol. Ecol. 22, 4369-4383. https://doi.org/10.1111/mec.12413.

Chan, J.D., Zhang, D., Liu, X., Zarowiecki, M.Z., Berriman, M., Marchant, J.S., 2016. Data in brief dataset for a Dugesia japonica de novo transcriptome assembly, utilized for defining the voltage-gated like ion channel superfamily. Data Br. 9, 1044-1047. https://doi.org/10.1016/j.dib.2016.11.022

Darriba, D., Taboada, G.L., Doallo, R., Posada, D., 2012. jModelTest 2: more models, new heuristics and parallel computing. 772-772. Nat. Methods 9. https://doi.org/10. 1038/nmeth.2109.

Dayrat, B., 2005. Towards integrative taxonomy. Biol. J. Linn. Soc. 407-415.

de Queiroz, K., 2007. Species concepts and species delimitation. Syst Biol 56, 879-886. https://doi.org/10.1080/10635150701701083.

de Queiroz, K., 1998. The general linage concept of species, species criteria, and the process of speciation. A conceptual unification and terminological recommendations. In: Endless Forms: Species and Speciation. Oxford University Press, pp. 57-75. https://doi.org/10.1098/rspb.1993.0016.

De Vries, E.J., 1988a. A synopsis of the nominal species of the subgenus Dugesia (Platyhelminthes: Tricladida: Paludicola) from Africa and Madagascar. Zool. J. Linn. Soc. 92, 345-382. https://doi.org/10.1111/j.1096-3642.1988.tb01729.x.

De Vries, E.J., 1988b. Further contributions to the taxonomy and biogeography of the subgenus Dugesia (Platyhelminthes: Tricladida: Paludicola) in the Mediterranean region and the Middle East. Isr. J. Zool. 35, 109-136. https://doi.org/10.1080/ 00212210.1988.10688607.

De Vries, E.J., 1986a. On the taxonomic status of Dugesia gonocephala and Dugesia subtentaculata (Turbellaria, Tricladida, Paludicola). J. Zool. London 43-59.

De Vries, E.J., 1986b. On the karyology of Dugesia gonocephala s.l. (Turbellaria, Tricladida) from Montpellier, France. Hydrobiologia 251-256.

Dejaco, T., Gassner, M., Arthofer, W., Schlick-Steiner, B.C., Steiner, F.M., 2016. Taxonomist's nightmare evolutionist's delight: An integrative approach resolves species limits in jumping bristletails despite widespread hybridization and parthenogenesis. Syst. Biol. 65, 947-974. https://doi.org/10.1093/sysbio/syw003.

Drummond, A.J., Suchard, M.A., Xie, D., Rambaut, A., 2012. Bayesian phylogenetics with BEAUti and the BEAST 1.7. Mol. Biol. Evol. 29, 1969-1973. https://doi.org/10.1093/ molbev/mss075.

Egea, E., David, B., Choné, T., Laurin, B., Féral, J.P., Chenuil, A., 2016. Morphological and genetic analyses reveal a cryptic species complex in the echinoid Echinocardium cordatum and rule out a stabilizing selection explanation. Mol. Phylogenet. Evol. 94, 207-220. https://doi.org/10.1016/j.ympev.2015.07.023.

Ence, D.D., Carstens, B.C., 2011. SpedeSTEM: A rapid and accurate method for species delimitation. Mol. Ecol. Resour. 11, 473-480. https://doi.org/10.1111/j.1755-0998. 2010.02947.x.

Ezard, T., Fujisawa, T., Barraclough, T., 2009. splits: SPecies' LImits by Threshold Statistics. R package version 1.0-11/r29. Retrieved from http://R-Forge.R-project. org/projects/splits/, accessed February 2018

Faria, R., Navarro, A., 2010. Chromosomal speciation revisited: rearranging theory with pieces of evidence. Trends Ecol. Evol. 25, 660-669. https://doi.org/10.1016/j.tree. 2010.07.008.

Feschotte, C., Pritham, E.J., 2007. DNA Transposons and the evolution of eukaryotic genomes. Annu. Rev. Genet. 331-368.

Fišer, C., Robinson, C.T., Malard, F., 2018. Cryptic species as a window into the paradigm shift of the species concept. Mol. Ecol. 27, 613-635. https://doi.org/10.1111/mec. 14486.

Fisher, R.A., 1999. The genetical theory of natural selection. Oxford University Press, New York.

Flot, J.-F., 2015. Species delimitation' s coming of age. Syst. Biol. 64, 897-899. https:// doi.org/10.1093/sysbio/syv071.

Frankham, R., Ballou, J.D., Dudash, M.R., Eldridge, M.D.B., Fenster, C.B., Lacy, R.C., Mendelson, J.R., Porton, I.J., Ralls, K., Ryder, O.A., 2012. Implications of different species concepts for conserving biodiversity. Biol. Conserv. 153, 25-31. https://doi. org/10.1016/j.biocon.2012.04.034.

Garcia-Cisneros, A., Pérez-Portela, R., Almroth, B.C., Degerman, S., Palacín, C., Sköld, H.N., 2015. Long telomeres are associated with clonality in wild populations of the fissiparous starfish Coscinasterias tenuispina. Heredity (Edinb). 115, 437-443. https:// doi.org/10.1038/hdy.2015.43.

Garcia-Fernàndez, J., Bayascas-Ramírez, J.R., Marfany, G., Muñoz-Mármol, A.M., Casali, A., Baguñà, J., Saló, E., 1995. High copy number of highly similar mariner-like transposons in planarian (Platyhelminthe): Evidence for a trans-phyla horizontal transfer. Mol. Biol. Evol. 12, 421-431.

Gernhard, T., Hartmann, K., Steel, M., 2008. Stochastic properties of generalised Yule models, with biodiversity applications. J. Math. Biol. 57, 713-735. https://doi.org/ 10.1007/s00285-008-0186-y.

Gourbault, N., 1981. The karyotypes of Dugesia species from Spain (Turbellaria, Tricladida). Hydrobiologia 84, 45-52. https://doi.org/10.1007/BF00026162.

Gourbault, N., Benazzi, M., 1979. Une nouvelle espèce ibérique du "groupe Dugesia gonocephala" (Turbellariés, Triclades). Bull. du Muséum Natl. d'histoire Nat Paris 2, 329-337.

Gourbault, N., Benazzi, M., 1975. Karyological data on some species of the genus Cura (Tricladida, Paludicola). Can. J. Genet. Cytol. 17, 345-354.

Grohme, M.A., Schloissnig, S., Rozanski, A., Pippel, M., Young, G.R., Winkler, S., Brandl, H., Henry, I., Dahl, A., Powell, S., Hiller, M., Myers, E., Rink, J.C., 2018. The genome of Schmidtea mediterranea and the evolution of core cellular mechanisms. Nature 554, 56-61. https://doi.org/10.1038/nature25473.

Heled, J., Drummond, A.J., 2010. Bayesian inference of species trees from multilocus data. Mol. Biol. Evol. 27, 570-580. https://doi.org/10.1093/molbev/msp274.

IUCN, 2012. IUCN Red List categories and criteria, Second. ed, IUCN Bulletin. Gland, Switzerland and Cambridge. https://doi.org/10.9782-8317-0633-5.

Kapli, P., Lutteropp, S., Zhang, J., Kobert, K., Pavlidis, P., Stamatakis, A., Flouri, T., 2017. Multi-rate Poisson tree processes for single-locus species delimitation under maximum likelihood and Markov chain Monte Carlo. Bioinformatics 33, 1630-1638. https://doi.org/10.1093/bioinformatics/btx025.

Katoh, K., Standley, D.M., 2013. MAFFT multiple sequence alignment software version $7:$ Improvements in performance and usability. Mol. Biol. Evol. 30, 772-780. https:// doi.org/10.1093/molbev/mst010.

Kawakatsu, M., Oki, I., Tamura, S., Sugino, H., 1976. Studies on the morphology, karyology and taxonomy of the Japanese freshwater planarian Dugesia japonica Ichikawa et Kawakatsu, with a description of a new subspecies, Dugesia japonica ryukyuensis subspec. nov. Bull. Fuji Women's Coll. 14, 81-126.

Knakievicz, T., 2014. Planarians as invertebrate bioindicators in freshwater environmental quality: the biomarkers approach. Ecotoxicol. Environ. Contam. 9, 1-12. https://doi.org/10.5132/eec.2014.01.001.

Knowles, L.L., Carstens, B.C., 2007. Delimiting species without monophyletic gene trees. Syst. Biol. 56, 887-895. https://doi.org/10.1080/10635150701701091.

Lázaro, E.M., Harrath, A.H., Stocchino, G.a., Pala, M., Baguñà, J., Riutort, M., 2011 Schmidtea mediterranea phylogeography: an old species surviving on a few Mediterranean islands? BMC Evol. Biol. 11, 274. https://doi.org/10.1186/1471 2148-11-274.

Lázaro, E.M., Sluys, R., Pala, M., Stocchino, G.A., Baguñà, J., Riutort, M., 2009. Molecular barcoding and phylogeography of sexual and asexual freshwater planarians of the genus Dugesia in the Western Mediterranean (Platyhelminthes, Tricladida, 
Dugesiidae). Mol. Phylogenet. Evol. 52, 835-845. https://doi.org/10.1016/j.ympev. 2009.04.022.

Lemmon, A.R., Brown, J.M., Stanger-Hall, K., Lemmon, E.M., 2009. The effect of ambiguous data on phylogenetic estimates obtained by maximum likelihood and bayesian inference. Syst. Biol. 58, 130-145. https://doi.org/10.1093/sysbio/syp017.

Leria, L., Sluys, R., Riutort, M., 2018. Diversification and biogeographic history of the Western Palearctic freshwater flatworm genus Schmidtea (Tricladida: Dugesiidae), with a redescription of Schmidtea nova. J. Zool. Syst. Evol. Res. 56, 335-351. https:// doi.org/10.1111/jzs.12214.

Leria, L., Vila-Farré, M., Solà, E., Riutort, M., 2019. Outstanding intraindividual genetic diversity in fissiparous planarians (Dugesia, Platyhelminthes) with facultative sex. BMC Evol. Biol. https://doi.org/10.1186/s12862-019-1440-1. in press.

Levan, A., Fredga, K., Sandberg, A.A., 1964. Nomenclature for centromeric position on chromosomes. Hereditas 52, 201-220. https://doi.org/10.1111/j.1601-5223.1964. tb01953.x.

Li, H., Durbin, R., 2009. Fast and accurate short read alignment with Burrows-Wheeler transform. Bioinformatics 25 (14), 1754-1760. https://doi.org/10.1093/ bioinformatics/btp324.

Lunter, G., Goodson, M., 2011. Stampy: A statistical algorithm for sensitive and fast mapping of Illumina sequence reads. Genome Res. 21, 936-939. https://doi.org/10. 1101/gr.111120.110.

Luo, A., Ling, C., Ho, S.Y.W., Chao-dong, Z., 2018. Comparison of methods for molecular species delimitation across a range of speciation scenarios. Syst. Biol. 67, 830-846. https://doi.org/10.1093/sysbio/syy011.

Mateos, E., Sluys, R., Riutort, M., Álvarez-Presas, M., 2017. Species richness in the genus Microplana (Platyhelminthes, Tricladida, Microplaninae) in Europe: As yet no asymptote in sight. Invertebr. Syst. 31, 269-301. https://doi.org/10.1071/IS16038.

Mayr, E., 1942. Systematics and the origin of species from the viewpoint of a zoologist. Harvard University Press, Cambridge, MA, London.

Miller, M.A., Pfeiffer, W., Schwartz, T., 2010. Creating the CIPRES Science Gateway for inference of large phylogenetic trees. 2010 Gatew. Comput. Environ. Work. GCE 2010. https://doi.org/10.1109/GCE.2010.5676129.

Obertegger, U., Cieplinski, A., Fontaneto, D., Papakostas, S., 2018. Mitonuclear discordance as a confounding factor in the DNA taxonomy of monogonont rotifers. Zool. Scr. 47, 122-132. https://doi.org/10.1111/zsc.12264.

Padial, J.M., Miralles, A., 2010. The integrative future of taxonomy. Front. Zool. 7, 16. https://doi.org/10.1186/1742-9994-7-16.

Pala, M., Casu, S., Stocchino, G., 1999. Karyology and karyotype analysis of diploid freshwater planarian populations of the Dugesia gonocephala group (Platyhelminthes, Tricladida) found in Sardinia. Hydrobiologia 392, 113-119.

Pala, M., Casu, S., Vacca, R.A., 1981. Dugesia hepta, nuova specie di Planaria di acqua dolce di Sardegna appartenente alla superspecie Dugesia gonocephala (Dugès) (Turbellaria, Tricladida). Boll. della Soc. Sarda di Sci. Nat. 20, 97-107.

Pala, M., Stocchino, G.A., Corso, G., Casu, S., Pala, M., Stocchino, G.A., Corso, G., Pala, M., 2000. A new species of freshwater planarian belonging to the genus Dugesia (Platyhelminthes, Tricladida) from Sardinia. Ital. J. Zool. 67, 379-383. https://doi. org/10.1080/11250000009356343.

Pante, E., Puillandre, N., Viricel, A., Arnaud-Haond, S., Aurelle, D., Castelin, M., Chenuil, A., Destombe, C., Forcioli, D., Valero, M., Viard, F., Samadi, S., 2015a. Species are hypotheses: Avoid connectivity assessments based on pillars of sand. Mol. Ecol. 24, 525-544. https://doi.org/10.1111/mec.13048.

Pante, E., Schoelinck, C., Puillandre, N., 2015b. From integrative taxonomy to species description: One step beyond. Syst. Biol. 64, 152-160. https://doi.org/10.1093/ sysbio/syu083.

Papakostas, S., Michaloudi, E., Proios, K., Brehm, M., Verhage, L., Rota, J., Peña, C., Stamou, G., Pritchard, V.L., Fontaneto, D., Declerck, S.A.J., 2016. Integrative taxonomy recognizes evolutionary units despite widespread mitonuclear discordance: Evidence from a rotifer cryptic species complex. Syst. Biol. 65, 508-524. https://doi. org/10.1093/sysbio/syw016.

Pérez-Ponce de León, G., Poulin, R., 2016. Taxonomic distribution of cryptic diversity among metazoans: not so homogeneous after all. Biol Lett 12.

Pongratz, N., Storhas, M., Carranza, S., Michiels, N.K., 2003. Phylogeography of competing sexual and parthenogenetic forms of a freshwater flatworm: patterns and explanations. BMC Evol. Biol. 3, 23. https://doi.org/10.1186/1471-2148-3-23.

Pons, J., Barraclough, T.G., Gomez-Zurita, J., Cardoso, A., Duran, D.P., Hazell, S., Kamoun, S., Sumlin, W.D., Vogler, A.P., 2006. Sequence-based species delimitation for the DNA taxonomy of undescribed insects. Syst. Biol. 55, 595-609. https://doi. org/10.1080/10635150600852011.

Puillandre, N., Lambert, A., Brouillet, S., Achaz, G., 2012. ABGD, Automatic Barcode Gap Discovery for primary species delimitation. Mol. Ecol. 21, 1864-1877. https://doi. org/10.1111/j.1365-294X.2011.05239.x.

Rader, R.B., Unmack, P.J., Moore, J.N., 2017. Population genetics and dispersal of the flatworm, Polycelis coronata: a test of the habitat stability hypothesis. J. Freshw. Ecol. 32, 1-14. https://doi.org/10.1080/02705060.2016.1253624.

Rambaut, A., Drummond, A.J., Xie, D., Baele, G., Suchard, M.A., 2018. Posterior summarisation in Bayesian phylogenetics using Tracer 1.7. Syst. Biol. 67 (5), 901-904. https://doi.org/10.1093/sysbio/syy032.

Rannala, B., Yang, Z., 2003. Bayes estimation of species divergence times and ancestral population sizes using DNA sequences from multiple loci. Genetics 164, 1645-1656. https://doi.org/10.1073/pnas.0913022107.

Razkin, O., Gómez-Moliner, B.J., Vardinoyannis, K., Martínez-Ortí, A., Madeira, M.J., 2017. Species delimitation for cryptic species complexes: case study of Pyramidula (Gastropoda, Pulmonata). Zool. Scr. 46, 55-72. https://doi.org/10.1111/zsc.12192.

Ribas, M., 1990. Cariologia, sistemàtica i biogeografia de les Planàries d'aigües dolces als Països Catalans. PhD Thesis. Universitat de Barcelona.

Robb, S., Gotting, K., Ross, E., Sánchez Alvarado, A., 2015. SmedGD 2.0: The Schmidtea mediterranea genome database. Genesis 70, 773-779. https://doi.org/10.1002/dvg. 22872.

Ronquist, F., Teslenko, M., Van Der Mark, P., Ayres, D.L., Darling, A., Höhna, S., Larget, B., Liu, L., Suchard, M.A., Huelsenbeck, J.P., 2012. Mrbayes 3.2: Efficient bayesian phylogenetic inference and model choice across a large model space. Syst. Biol. 61, 539-542. https://doi.org/10.1093/sysbio/sys029.

Sáez, A.G., Probert, I., Geisen, M., Quinn, P., Young, J.R., Medlin, L.K., 2003. Pseudocryptic speciation in coccolithophores. Proc. Natl. Acad. Sci. 100, 7163-7168. https://doi.org/10.1073/pnas.1132069100.

Sakai, M., Sakaizumi, M., 2012. The Complete Mitochondrial Genome of Dugesia japonica (Platyhelminthes; Order Tricladida). Zoolog. Sci. 29, 672-680. https://doi.org/10. 2108/zsj.29.672.

Sambrook, J., Fritschi, E.F., Maniatis, T., 1989. Molecular cloning: A laboratory manual. Cold Spring Harbor Laboratory Press, New York.

Schlick-Steiner, B.C., Steiner, F.M., Seifert, B., Stauffer, C., Christian, E., Crozier, R.H., 2010. Integrative taxonomy: A multisource approach to exploring biodiversity. Annu. Rev. Entomol. 55, 421-438. https://doi.org/10.1146/annurev-ento-112408-085432.

Silva, P.G., Goy, J.L., Zazo, C., Giménez, J., Fornós, J., Cabero, A., Bardají, T., Mateos, R., González-Hernández, F.M., Hillaire-Marcel, C., Bassam, G., 2005. Mallorca island: Geomorphological evolution and neotectonics, in: Sixth International Conference on Geomorphology. https://doi.org/10.15713/ins.mmj.3.

Sluys, R., Hazevoet, C., 1999. Pluralism in species concepts: Dividing nature at its diverse joints. Species Divers. 243-256. https://doi.org/10.12782/specdiv.4.243.

Sluys, R., Kawakatsu, M., De León, R.P., 2005. Morphological stasis in an old and widespread group of species: Contribution to the taxonomy and biogeography of the genus Girardia (Platyhelminthes, Tricladida, Paludicola). Stud. Neotrop. Fauna Environ. 40, 155-180. https://doi.org/10.1080/01650520500070220.

Sluys, R., Solà, E., Gritzalis, K., Vila-Farré, M., Mateos, E., Riutort, M., 2013. Integrative delineation of species of Mediterranean freshwater planarians (Platyhelminthes: Tricladida: Dugesiidae). Zool. J. Linn. Soc. 169, 523-547. https://doi.org/10.1111/ zoj.12077.

Smith, K.L., Harmon, L.J., Shoo, L.P., Melville, J., 2011. Evidence of constrained phenotypic evolution in a cryptic species complex of agamid lizards. Evolution (N. Y) 65, 976-992. https://doi.org/10.1111/j.1558-5646.2010.01211.x.

Solà, E., 2014. Systematics and historical biogeography of the genus Dugesia (Platyhelminthes, Tricladida). PhD Thesis. University of Barcelona.

Solà, E., Sluys, R., Gritzalis, K., Riutort, M., 2013. Fluvial basin history in the northeastern Mediterranean region underlies dispersal and speciation patterns in the genus Dugesia (Platyhelminthes, Tricladida, Dugesiidae). Mol. Phylogenet. Evol. 66, 877-888. https://doi.org/10.1016/j.ympev.2012.11.010.

Souza, S., Morais, A.L., Bichuette, M.E., Leal-Zanchet, A., 2016. Two new species of freshwater flatworms (Platyhelminthes: Tricladida: Continenticola) from South American caves. Zootaxa 4092, 107-121.

Stamatakis, A., 2006. RAxML-VI-HPC: Maximum likelihood-based phylogenetic analyses with thousands of taxa and mixed models. Bioinformatics 22, 2688-2690. https:// doi.org/10.1093/bioinformatics/btl446.

Stocchino, G., Manconi, R., 2013. Overview of life cycles in model species of the genus Dugesia (Platyhelminthes: Tricladida). Ital. J. Zool. 80, 319-328. https://doi.org/10. 1080/11250003.2013.822025.

Stocchino, G.A., Manconi, R., Corso, G., Pala, M., 2004. Karyology and karyometric analysis of an Afrotropical freshwater planarian (Platyhelminthes, Tricladida). Ital. J. Zool. 71, 89-93. https://doi.org/10.1080/11250000409356557.

Stocchino, G.A., Manconi, R., Corso, G., Sluys, R., Casu, S., Pala, M., 2009. African planarians: Morphology and karyology of Dugesia maghrebiana sp. n. (Platyhelminthes, Tricladida) from Tunisia. Ital. J. Zool. 76, 83-91. https://doi.org/10.1080/ 11250000802141683.

Stocchino, G.A., Sluys, R., Riutort, M., Solà, E., Manconi, R., 2017. Freshwater planarian diversity (Platyhelminthes: Tricladida: Dugesiidae) in Madagascar: New species, cryptic species, with a redefinition of character states. Zool. J. Linn. Soc. 181, 727-756. https://doi.org/10.1093/zoolinnean/zlx017.

Struck, T.H., Feder, J.L., Bendiksby, M., Birkeland, S., Cerca, J., Gusarov, V.I., Kistenich, S., Larsson, K.H., Liow, L.H., Nowak, M.D., Stedje, B., Bachmann, L., Dimitrov, D., 2018. Finding evolutionary processes hidden in cryptic species. Trends Ecol. Evol. 33, 153-163. https://doi.org/10.1016/j.tree.2017.11.007.

Talavera, G., Dincǎ, V., Vila, R., 2013. Factors affecting species delimitations with the GMYC model: Insights from a butterfly survey. Methods Ecol. Evol. 4, 1101-1110. https://doi.org/10.1111/2041-210X.12107.

Tamura, K., Peterson, D., Peterson, N., Stecher, G., Nei, M., Kumar, S., 2011. MEGA5: Molecular evolutionary genetics analysis using maximum likelihood, evolutionary distance, and maximum parsimony methods. Mol. Biol. Evol. 28, 2731-2739. https:// doi.org/10.1093/molbev/msr121.

Tamura, S., Oki, I., Kawakatsu, M., 1991. Karyological and taxonomic studies of Dugesia japonica from the Southwest Islands of Japan-II. Hydrobiologia 227, 157-162. https://doi.org/10.1007/BF00027596.

Tamura, S., Yamamoto, K., Takai, M., Oki, I., Kawakatsu, M., 1998. Karyology and biogeography of Dugesia japonica and Dugesia ryukyuensis in Kyushu, southern Japan. Hydrobiologia 383, 321-327. https://doi.org/10.1023/A:1003471207447.

Teal, J.M., 1957. Community metabolism in a temperate cold spring. Ecol. Monogr. 27, 283-302.

Tilly, L.J., 1968. The structure and dynamics of Cone spring. Ecol. Monogr. 38, 169-197.

Vacca, R.A., Casu, S., Pala, M., 1993. Popolamento planariologico dei fiumi del nord Sardegna: 2. I cariotipi dei tricladi d'acqua dolce rinvenuti nel bacino idrografico del fiume Coghinas. Boll. della Soc Sarda di Sci. Nat. 29, 59-73.

Van Steenkiste, N.W.L., Herbert, E.R., Leander, B.S., 2018. Species diversity in the marine microturbellarian Astrotorhynchus bifidus sensu lato (Platyhelminthes : Rhabdocoela) from the Northeast Pacific Ocean. Mol. Phylogenet. Evol. 120, 259-273. https://doi. 
org/10.1016/j.ympev.2017.12.012

Vandel, A., 1925. Planaria subtentaculata Drap. n'est qu'une race asexuee de Planaria gonocephala Duges. Bull. Biol. Fr. Belg. 59, 498-507.

Winsor, L., Sluys, R., 2018. Basic histological techniques for planarians. In: Rink, J.C (Ed.), Planarian regeneration: Methods and protocols. Springer Science + Business Media.

Xia, X., Lemey, P., 2009. Assessing substitution saturation with DAMBE. In: Lemey, P., Salemi, M., Vandamme, A.-.M. (Eds.), The Phylogenetic Handbook: A Practical Approach to DNA and Protein Phylogenetic Analysis and Hypothesis Testing. Cambridge University Press, pp. 611-626.

Xia, X., Xie, Z., 2001. DAMBE: Software package for data analysis in molecular biology and evolution. J. Hered. 371-373.
Xia, X., Xie, Z., Salemi, M., Chen, L., Wang, Y., 2003. An index of substitution saturation and its application. Mol. Phylogenet. Evol. 26, 1-7. https://doi.org/10.1016/S10557903(02)00326-3.

Yang, Z., 2015. The BPP program for species tree estimation and species delimitation. Curr. Zool. 61, 854-865. https://doi.org/10.1093/czoolo/61.5.854.

Yang, Z., Rannala, B., 2010. Bayesian species delimitation using multilocus sequence data. Proc. Natl. Acad. Sci. 107, 9264-9269. https://doi.org/10.1073/pnas. 0913022107.

Zhang, J., Kapli, P., Pavlidis, P., Stamatakis, A., 2013. A general species delimitation method with applications to phylogenetic placements. Bioinformatics 29, 2869-2876. https://doi.org/10.1093/bioinformatics/btt499. 